\title{
Structural and Co-Conformational Effects of Alkyne-Derived Subunits in Charged Donor-Acceptor [2]Catenanes
}

\author{
Ognjen Š. Miljanić, ${ }^{\dagger}$ William R. Dichtel, ${ }^{\dagger, \ddagger}$ Saeed I. Khan, ${ }^{\dagger}$ Shahab Mortezaei, ${ }^{\dagger}$ \\ James R. Heath, ${ }^{\ddagger}$ and J. Fraser Stoddart ${ }^{\dagger *}$ \\ † California NanoSystems Institute and Department of Chemistry and Biochemistry, \\ University of California, Los Angeles, 405 Hilgard Avenue, Los Angeles, California 90095 \\ † Division of Chemistry and Chemical Engineering, California Institute of Technology, \\ 1200 East California Boulevard, Pasadena, CA 91125
}

\section{Supporting Information} REVISED VERSION

\begin{tabular}{|c|}
\hline Correspondence Address \\
\hline Professor J Fraser Stoddart \\
California NanoSystems Institute and \\
Department of Chemistry and Biochemistry \\
University of California, Los Angeles \\
405 Hilgard Avenue \\
Los Angeles, CA 90095-1569 (USA) \\
Tel: (+1)-310-206-7078 \\
Fax: (+1)-310-206-1843 \\
Email: $\underline{\text { stoddart@ chem.ucla.edu }}$ \\
\hline
\end{tabular}


General Methods. All reagents were purchased from commercial suppliers (Aldrich or Fisher) and used without further purification. Cyclobis(paraquat- $p$-phenylene) hexafluorophosphate $^{\mathrm{S} 1}$ (CBPQT $\left.\cdot 4 \mathrm{PF}_{6}\right)$, 1,5-bis[2-(2-hydroxyethoxy)ethoxy)] naphthalene ${ }^{\mathrm{S} 2}$ (6a), 1,5-bis[2-(2-(2-hydroxyethoxy)ethoxy)ethoxy]naphthalene ${ }^{\mathrm{S} 3}(\mathbf{6 b})$, 1,5-bis[2-(2-(2-(2hydroxyethoxy)ethoxy)ethoxy)ethoxy]naphthalene ${ }^{\mathrm{S} 3}(\mathbf{6 c})$ and mono-tosylates ${ }^{\mathrm{S} 4 \mathrm{~S} 5}$ of $\mathbf{6 a}$ (S1) and 6b were prepared according to literature procedures. Preparation and characterization of catenanes $\mathbf{1 b} \cdot 4 \mathrm{PF}_{6}$ and $\mathbf{2 b} \cdot 4 \mathrm{PF}_{6}$, and their precursors $\mathbf{7 b}, \mathbf{8 b}$, and $\mathbf{9 b}$ was reported in a previous publication. ${ }^{56}$ Thin layer chromatography (TLC) was performed on silica gel $60 \mathrm{~F}_{254}$ (E. Merck). Column chromatography was performed on silica gel 60F (Merck 9385, 0.040-0.063 mm). Melting points were recorded on an Electrothermal 9100 instrument in open capillary tubes and are uncorrected. Routine nuclear magnetic resonance (NMR) spectra were recorded at $25{ }^{\circ} \mathrm{C}$ on a Bruker Avance 400 and 500 spectrometers, with working frequencies of 400 and $500 \mathrm{MHz}$ for ${ }^{1} \mathrm{H}$, and 100 and 125 $\mathrm{MHz}$ for ${ }^{13} \mathrm{C}$ nuclei respectively. VT-NMR spectra were recorder on a Bruker Avance 500 spectrometer and temperature-calibrated using neat $\mathrm{MeOH}$ (for $T<295 \mathrm{~K}$ ) and ethylene glycol (for $T>295 \mathrm{~K}$ ). Chemical shifts are reported in ppm relative to the signals corresponding to the residual non-deuterated solvents $\left(\mathrm{CDCl}_{3}: \delta 7.26 \mathrm{ppm}, \mathrm{CD}_{3} \mathrm{CN}: \delta 1.93\right.$ ppm, DMF- $\left.d_{7}: \delta 8.01 \mathrm{ppm}\right)$. All ${ }^{13} \mathrm{C}$ spectra were recorded with the simultaneous decoupling of proton nuclei. Fast atom bombardment mass spectra were obtained on a JEOL JMS-600H high resolution mass spectrometer equipped with a FAB probe.

Scheme S1. Synthesis of DNP Azide Derivative 7a.

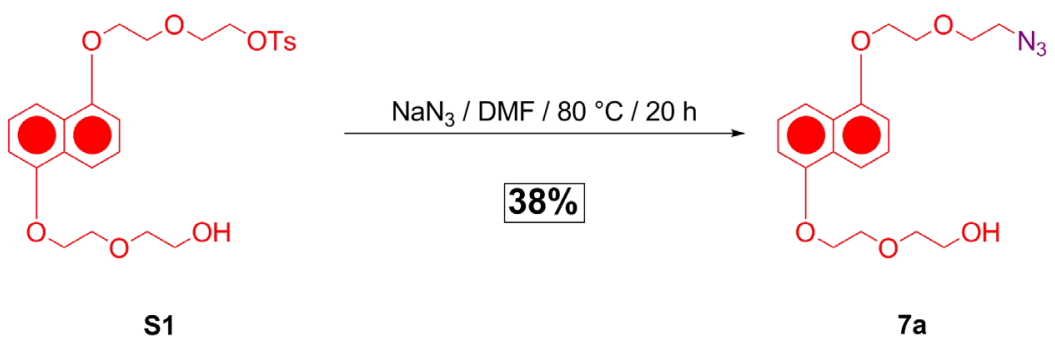

A solution of tosylate ${ }^{\mathrm{S} 4} \mathbf{S 1}(1.37 \mathrm{~g}, 2.78 \mathrm{mmol})$ and $\mathrm{NaN}_{3}(904 \mathrm{mg}, 13.9 \mathrm{mmol})$ in dry DMF (15 mL) was heated at $80{ }^{\circ} \mathrm{C}$ for $20 \mathrm{~h}$. After cooling, the solvent was removed in 
vacuo. The resulting brown oil was redissolved in $\mathrm{CH}_{2} \mathrm{Cl}_{2}$, and washed first with $1 \mathrm{M}$ aqueous $\mathrm{HCl}$ solution, followed by sat. aqueous $\mathrm{NaHCO}_{3}$ solution. After drying $\left(\mathrm{MgSO}_{4}\right)$ and removal of the solvent, the crude product was subjected to chromatography on silica $\left(\mathrm{EtOAc} \rightarrow\right.$ EtOAc/Me $\left.{ }_{2} \mathrm{CO}=50 / 50\right)$ to provide $380 \mathrm{mg}$ of $\mathbf{7 a}$ as a viscous yellow oil (38\%). 7a: MS (FAB+): $m / z$ (rel intensity) = $361\left(\mathrm{M}^{+}, 100 \%\right), 227$ (39), 160 (36), 149 (79). ${ }^{1} \mathrm{H}$ NMR $\left(500 \mathrm{MHz}, \mathrm{CDCl}_{3}\right): \delta 7.89\left(\mathrm{~d},{ }^{3} J(\mathrm{H}, \mathrm{H})=8.2 \mathrm{~Hz}, 1 \mathrm{H}\right), 7.87\left(\mathrm{~d},{ }^{3} J(\mathrm{H}, \mathrm{H})=8.2 \mathrm{~Hz}\right.$, $1 \mathrm{H}), 7.35\left(\mathrm{t},{ }^{3} J(\mathrm{H}, \mathrm{H})=8.2 \mathrm{~Hz}, 1 \mathrm{H}\right), 7.35\left(\mathrm{t},{ }^{3} J(\mathrm{H}, \mathrm{H})=8.2 \mathrm{~Hz}, 1 \mathrm{H}\right), 6.82\left(\mathrm{~d},{ }^{3} J(\mathrm{H}, \mathrm{H})=\right.$ $8.2 \mathrm{~Hz}, 1 \mathrm{H}), 6.80\left(\mathrm{~d},{ }^{3} J(\mathrm{H}, \mathrm{H})=8.2 \mathrm{~Hz}, 1 \mathrm{H}\right), 4.28-4.20(\mathrm{~m}, 4 \mathrm{H}), 3.96-3.90(\mathrm{~m}, 4 \mathrm{H}), 3.78-$ $3.76(\mathrm{~m}, 2 \mathrm{H}), 3.75-3.72$ (br m, 2H), 3.68-3.65 (m, 2H), 3.39 (t, $\left.{ }^{3} J(\mathrm{H}, \mathrm{H})=5.0 \mathrm{~Hz}, 2 \mathrm{H}\right)$, 2.62 (br s, 1H). $\left.{ }^{13} \mathrm{C} \mathrm{NMR} \mathrm{(125} \mathrm{MHz,} \mathrm{CDCl}_{3}\right): \delta 154.16(2 \mathrm{C}), 126.65,126.63,125.15$, $125.07,114.60,114.48,105.70,105.68,72.58,70.22$, 69.69, 69.58, 67.82, 67.76, 61.64, 50.67. HRMS Calcd for $\mathrm{C}_{18} \mathrm{H}_{23} \mathrm{~N}_{3} \mathrm{O}_{5}$ : 361.1638. Found: 361.1649 .

Scheme S2. Synthesis of DNP Monotosylate S2.

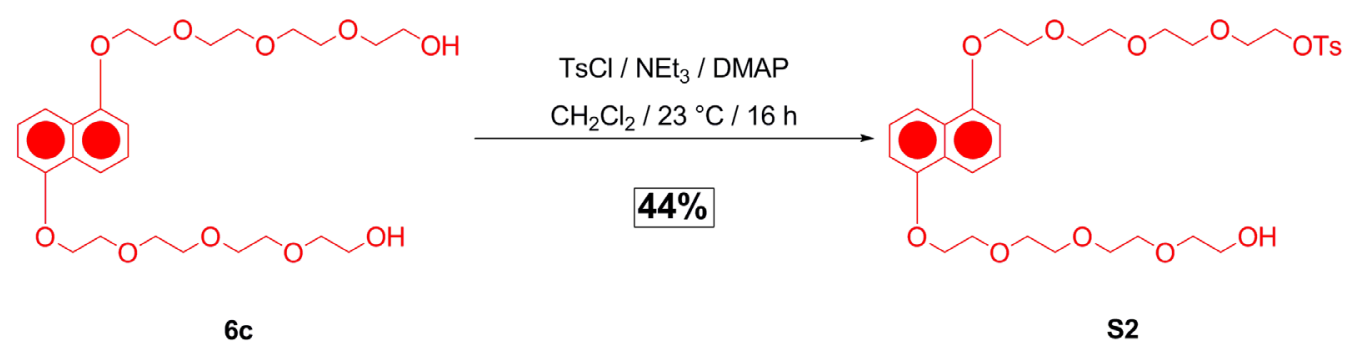

The diol 6c (1.38 g, $2.69 \mathrm{mmol}), \mathrm{NEt}_{3}(555 \mathrm{mg}, 780 \mu \mathrm{L}, 5.50 \mathrm{mmol})$, and DMAP (20 mg, $0.16 \mathrm{mmol})$ were dissolved in $\mathrm{CH}_{2} \mathrm{Cl}_{2}(50 \mathrm{~mL})$. A solution of $\mathrm{TsCl}(514 \mathrm{mg}, 2.71 \mathrm{mmol})$ in $\mathrm{CH}_{2} \mathrm{Cl}_{2}(18 \mathrm{~mL})$ was added dropwise to this mixture during $12 \mathrm{~h}$ at $23{ }^{\circ} \mathrm{C}$ (syringe pump, $1.5 \mathrm{~mL} / \mathrm{h}$ ). After the addition was complete, the reaction mixture was left to stir at $23{ }^{\circ} \mathrm{C}$ for 4 additional $\mathrm{h}$. It was then washed with sat. aqueous $\mathrm{NaHCO}_{3}$ solution and brine and dried $\left(\mathrm{MgSO}_{4}\right)$. Column chromatography $\left(\mathrm{CH}_{2} \mathrm{Cl}_{2} \rightarrow\right.$ EtOAc) isolated, in order of elution, the ditosylate ${ }^{\mathrm{S} 3}$ of $\mathbf{6 c}(310 \mathrm{mg}, 17 \%)$, followed by $\mathbf{S 2}(780 \mathrm{mg}, 44 \%)$, and unreacted starting material (400 mg, 29\%). S2: MS (FAB+): $\mathrm{m} / \mathrm{z}$ (rel intensity) $=666\left(\mathrm{M}^{+}, 100 \%\right) .{ }^{1} \mathrm{H}$ NMR (400 MHz, $\left.\mathrm{CDCl}_{3}\right): \delta 7.75\left(\mathrm{~d},{ }^{3} J(\mathrm{H}, \mathrm{H})=8.4 \mathrm{~Hz}, 1 \mathrm{H}\right), 7.74\left(\mathrm{~d},{ }^{3} J(\mathrm{H}, \mathrm{H})=8.4 \mathrm{~Hz}\right.$, $1 \mathrm{H}), 7.61\left(\mathrm{~d},{ }^{3} J(\mathrm{H}, \mathrm{H})=8.0 \mathrm{~Hz}, 2 \mathrm{H}\right), 7.21\left(\mathrm{t},{ }^{3} J(\mathrm{H}, \mathrm{H})=8.4 \mathrm{~Hz}, 1 \mathrm{H}\right), 7.21\left(\mathrm{t},{ }^{3} J(\mathrm{H}, \mathrm{H})=\right.$ 
$8.4 \mathrm{~Hz}, 1 \mathrm{H}), 7.12\left(\mathrm{~d},{ }^{3} J(\mathrm{H}, \mathrm{H})=8.0 \mathrm{~Hz}, 2 \mathrm{H}\right), 6.68\left(\mathrm{~d},{ }^{3} J(\mathrm{H}, \mathrm{H})=8.4 \mathrm{~Hz}, 1 \mathrm{H}\right), 6.68\left(\mathrm{~d},{ }^{3} J\right.$ $(\mathrm{H}, \mathrm{H})=8.4 \mathrm{~Hz}, 1 \mathrm{H}), 4.11-4.07(\mathrm{~m}, 4 \mathrm{H}), 3.97-3.94\left(\mathrm{t},{ }^{3} J(\mathrm{H}, \mathrm{H})=4.4 \mathrm{~Hz}, 2 \mathrm{H}\right), 3.81-3.77$ (m, 4H), 3.62-3.58 (m, 4H), 3.55-3.51 (m, 4H), 3.50-3.46 (m, 8H), 3.41-3.92 (m, 4H), $3.37-3.35$ (m, 2H), 3.13 (br s, $1 \mathrm{H}), 2.20(\mathrm{~s}, 3 \mathrm{H}) .{ }^{13} \mathrm{C} \mathrm{NMR}\left(100 \mathrm{MHz}, \mathrm{CDCl}_{3}\right): \delta 154.26$ (2C), 144.76, 132.84, 129.80, 127.78, 126.64 (2C), 125.11 (2C), 114.51 (2C), 105.64 (2C), 72.49, 70.76 (2C), 70.52, 70.49, 70.48, 70.45, 70.38, 70.16, 69.63 (2C), 69.37, 68.46, 67.83 (2C), 61.43, 21.45. HRMS Calcd for $\mathrm{C}_{33} \mathrm{H}_{46} \mathrm{O}_{12} \mathrm{~S}: 666.2710$. Found: 666.2693.

Scheme S3. Synthesis of DNP Azide Derivative 7c.

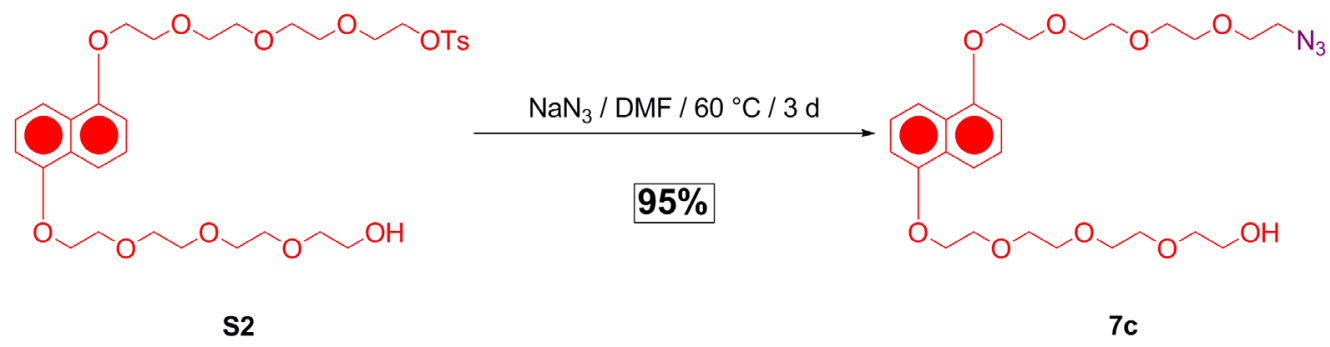

The tosylate $\mathbf{S 2}(780 \mathrm{mg}, 1.17 \mathrm{mmol})$ and $\mathrm{NaN}_{3}(130 \mathrm{mg}, 2.00 \mathrm{mmol})$ were dissolved in dry DMF $(50 \mathrm{~mL})$. The mixture was stirred at $60^{\circ} \mathrm{C}$ for $3 \mathrm{~d}$. After cooling, the solvent was removed in vacuo, and the crude material was filtered through a plug of silica, eluting with $\mathrm{Me}_{2} \mathrm{CO}$. A brown oil was obtained and found to be pure 7c (599 mg, 95\%). 7c: MS $(\mathrm{FAB}+): \mathrm{m} / z$ (rel intensity) $=537\left(\mathrm{M}^{+}, 100 \%\right), 510(28) .{ }^{1} \mathrm{H} \mathrm{NMR}\left(400 \mathrm{MHz}, \mathrm{CDCl}_{3}\right): \delta$ $7.79\left(\mathrm{br} \mathrm{d},{ }^{3} J(\mathrm{H}, \mathrm{H})=8.4 \mathrm{~Hz}, 2 \mathrm{H}\right), 7.27\left(\mathrm{br} \mathrm{t},{ }^{3} J(\mathrm{H}, \mathrm{H})=8.4 \mathrm{~Hz}, 2 \mathrm{H}\right), 6.75\left(\mathrm{br} \mathrm{d},{ }^{3} J(\mathrm{H}, \mathrm{H})=\right.$ $8.4 \mathrm{~Hz}, 2 \mathrm{H}), 4.20-4.18(\mathrm{~m}, 4 \mathrm{H}), 3.91-3.88(\mathrm{~m}, 4 \mathrm{H}), 3.73-3.70(\mathrm{~m}, 4 \mathrm{H}), 3.64-3.58(\mathrm{~m}$, 8H), 3.56-3.53 (m, 8H), 3.50-3.48 (m, 2H), 3.27-3.24 (m, 2H), 3.09 (br s, 1H). ${ }^{13} \mathrm{C} \mathrm{NMR}$ $\left(100 \mathrm{MHz}, \mathrm{CDCl}_{3}\right): \delta 154.30,154.27,126.71,126.70,125.13,125.11,114.60,114.55$, 105.70, 105.66, 72.54, 70.89, 70.81, 70.66, 70.58, 70.55, 70.50, 70.48, 70.16, 69.91, 69.71 (2C), 67.86 (2C), 61.47, 50.58. HRMS Calcd for $\mathrm{C}_{26} \mathrm{H}_{39} \mathrm{~N}_{3} \mathrm{O}_{9}$ : 537.2686. Found: 537.2684. 
Scheme S4. Synthesis of DNP Azidoalkyne Derivative 8a.

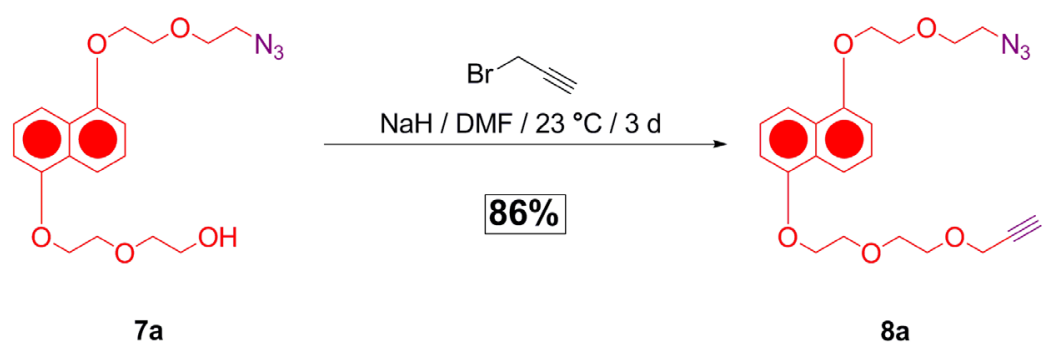

Compound 7a $(380 \mathrm{mg}, 1.05 \mathrm{mmol})$ was dissolved in dry DMF $(30 \mathrm{~mL})$ and treated with $\mathrm{NaH}$ (60 mg, $2.10 \mathrm{mmol})$. After the evolution of gas ceased ( 30 min), propargyl bromide ( $230 \mu \mathrm{L}$ of $80 \%$ wt solution in $\mathrm{PhMe}, 2.10 \mathrm{mmol}$ ) was added via a syringe. The mixture was left to stir at $23{ }^{\circ} \mathrm{C}$ for $3 \mathrm{~d}$. Solvent was removed in vacuo and the crude mixture was subjected to column chromatography $\left(\mathrm{CH}_{2} \mathrm{Cl}_{2} / \mathrm{MeOH}=95 / 5\right)$. The first fraction contained 8a as a brown oil $(350 \mathrm{mg}, 86 \%)$. 8a: ${ }^{1} \mathrm{H}$ NMR $\left(400 \mathrm{MHz}, \mathrm{CDCl}_{3}\right): \delta 7.88\left(\mathrm{~d},{ }^{3} J(\mathrm{H}, \mathrm{H})=\right.$ $8.4 \mathrm{~Hz}, 1 \mathrm{H}), 7.87\left(\mathrm{~d},{ }^{3} J(\mathrm{H}, \mathrm{H})=8.4 \mathrm{~Hz}, 1 \mathrm{H}\right), 7.37\left(\mathrm{t},{ }^{3} J(\mathrm{H}, \mathrm{H})=8.4 \mathrm{~Hz}, 2 \mathrm{H}\right), 6.85\left(\mathrm{~d},{ }^{3} J\right.$ $(\mathrm{H}, \mathrm{H})=8.4 \mathrm{~Hz}, 1 \mathrm{H}), 6.85\left(\mathrm{~d},{ }^{3} J(\mathrm{H}, \mathrm{H})=8.4 \mathrm{~Hz}, 1 \mathrm{H}\right), 4.29-4.27(\mathrm{~m}, 4 \mathrm{H}), 4.22\left(\mathrm{~d},{ }^{4} J(\mathrm{H}, \mathrm{H})\right.$ $=2.5 \mathrm{~Hz}, 2 \mathrm{H}), 3.99-3.96(\mathrm{~m}, 4 \mathrm{H}), 3.82-3.77(\mathrm{~m}, 6 \mathrm{H}), 3.41\left(\mathrm{t},{ }^{3} \mathrm{~J}(\mathrm{H}, \mathrm{H})=5.0 \mathrm{~Hz}, 4 \mathrm{H}\right), 2.44$ $\left(\mathrm{t},{ }^{4} J(\mathrm{H}, \mathrm{H})=2.5 \mathrm{~Hz}, 1 \mathrm{H}\right) .{ }^{13} \mathrm{C}$ NMR $\left(100 \mathrm{MHz}, \mathrm{CDCl}_{3}\right): \delta 154.35,154.26,126.77(2 \mathrm{C})$, $125.20,125.12,114.77,114.58,105.79,105.75,79.69,74.66,70.77,70.37,69.84$ (2C), $69.22,67.96,67.94,58.47,50.82$.

Scheme S5. Synthesis of DNP Azidoalkyne Derivative 8c.
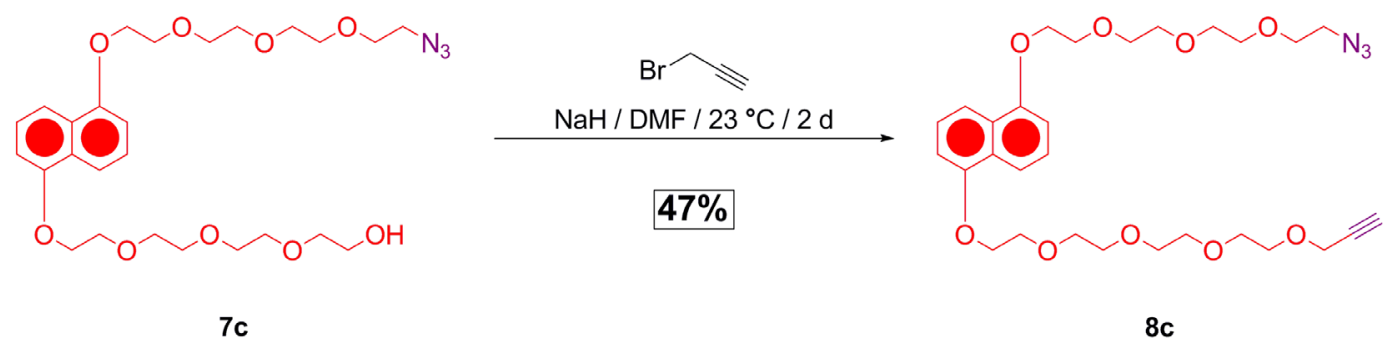

Compound 7c (599 mg, $1.12 \mathrm{mmol})$ was dissolved in dry DMF $(25 \mathrm{~mL})$ and treated with $\mathrm{NaH}$ (48 mg, $2.00 \mathrm{mmol}$ ). After the evolution of gas ceased ( 30 min), propargyl bromide (334 $\mu \mathrm{L}$ of $80 \%$ wt solution in $\mathrm{PhMe}, 3.00 \mathrm{mmol}$ ) was added via a syringe. The mixture was stirred for $2 \mathrm{~d}$ at $23{ }^{\circ} \mathrm{C}$ and subsequently quenched with $\mathrm{MeOH}(2.0 \mathrm{~mL})$. Solvents 
were removed in vacuo and the resulting crude mixture was filtered through a plug of silica (eluting with $\left.\mathrm{Me}_{2} \mathrm{CO}\right)$, and subsequently subjected to chromatography $\left(\mathrm{CH}_{2} \mathrm{Cl}_{2} / \mathrm{Me}_{2} \mathrm{CO}=\right.$ 75/25) to provide pure 8c as the second fraction (300 mg, 47\%). 8c: MS (FAB+): $\mathrm{m} / z$ (rel intensity) $=575\left(\mathrm{M}^{+}, 94 \%\right), 548(28), 537$ (100), 510 (30). ${ }^{1} \mathrm{H}$ NMR (500 MHz, $\left.\mathrm{CDCl}_{3}\right): \delta$ $7.86\left(\right.$ br d, $\left.{ }^{3} J(\mathrm{H}, \mathrm{H})=8.4 \mathrm{~Hz}, 2 \mathrm{H}\right), 7.34\left(\mathrm{t},{ }^{3} J(\mathrm{H}, \mathrm{H})=8.4 \mathrm{~Hz}, 2 \mathrm{H}\right), 6.83\left(\mathrm{~d},{ }^{3} J(\mathrm{H}, \mathrm{H})=8.4\right.$ $\mathrm{Hz}, 2 \mathrm{H}), 4.29\left(\mathrm{t},{ }^{3} J(\mathrm{H}, \mathrm{H})=5.0 \mathrm{~Hz}, 4 \mathrm{H}\right), 4.18\left(\mathrm{~d},{ }^{4} J(\mathrm{H}, \mathrm{H})=2.5 \mathrm{~Hz}, 2 \mathrm{H}\right), 3.99\left(\mathrm{t},{ }^{3} J(\mathrm{H}, \mathrm{H})\right.$ $=5.0 \mathrm{~Hz}, 4 \mathrm{H}), 3.81-3.77(\mathrm{~m}, 4 \mathrm{H}), 3.71-3.67(\mathrm{~m}, 4 \mathrm{H}), 3.67-3.63(\mathrm{~m}, 14 \mathrm{H}), 3.34\left(\mathrm{t},{ }^{3} J\right.$ $(\mathrm{H}, \mathrm{H})=5.0 \mathrm{~Hz}, 4 \mathrm{H}), 2.41\left(\mathrm{t},{ }^{4} J(\mathrm{H}, \mathrm{H})=2.5 \mathrm{~Hz}, 1 \mathrm{H}\right) .{ }^{13} \mathrm{C} \mathrm{NMR}\left(125 \mathrm{MHz}, \mathrm{CDCl}_{3}\right): \delta$ 154.25 (2C), 126.69 (2C), 124.95 (2C), 114.53 (2C), 105.58 (2C), 79.58, 74.37, 70.90, 70.88, 70.68, 70.62 (2C), 70.60, 70.54, 70.51, 70.29, 69.89, 69.71 (2C), 69.00, 67.83 (2C), 58.27, 50.56. HRMS Calcd for $\mathrm{C}_{29} \mathrm{H}_{41} \mathrm{~N}_{3} \mathrm{O}_{9}:$ 575.2842. Found: 575.2826.

Scheme S6. Synthesis of DNP Diyne Derivative 9a.

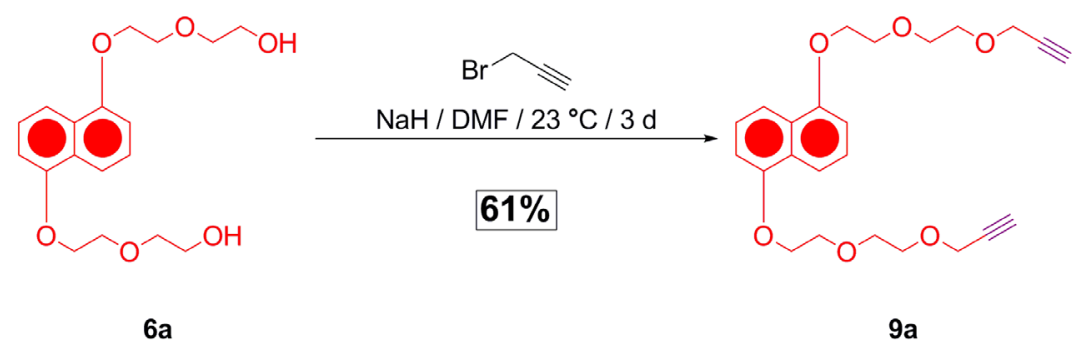

Diol 6a (1.12 g, $3.33 \mathrm{mmol})$ was dissolved in dry DMF (25 mL) and treated with NaH (192 mg, $8.00 \mathrm{mmol})$. After the evolution of gas ceased ( 30 min), propargyl bromide (2.2 $\mathrm{mL}$ of $80 \%$ wt solution in $\mathrm{PhMe}, 20.0 \mathrm{mmol}$ ) was added via a syringe. The mixture was stirred at $23{ }^{\circ} \mathrm{C}$ for $3 \mathrm{~d}$. $\mathrm{MeOH}(2.0 \mathrm{~mL})$ was added and the solvents were removed in vacuo. Crude brown oil was filtered through a plug of silica, eluting with $\mathrm{Me}_{2} \mathrm{CO}$, to provide pure 9a (840 mg, 61\%) as a dark yellow oil which solidifies on standing, mp 85$88{ }^{\circ}$ C. 9a: $\mathrm{MS}(\mathrm{FAB}+): \mathrm{m} / z$ (rel intensity) = $412\left(\mathrm{M}^{+}, 100 \%\right), 374(16) .{ }^{1} \mathrm{H} \mathrm{NMR}(400$ $\left.\mathrm{MHz}_{\mathrm{CDCl}}\right): \delta 7.74\left(\mathrm{~d},{ }^{3} J(\mathrm{H}, \mathrm{H})=8.4 \mathrm{~Hz}, 2 \mathrm{H}\right), 7.21\left(\mathrm{t},{ }^{3} J(\mathrm{H}, \mathrm{H})=8.4 \mathrm{~Hz}, 2 \mathrm{H}\right), 6.68(\mathrm{~d}$, $\left.{ }^{3} J(\mathrm{H}, \mathrm{H})=8.4 \mathrm{~Hz}, 2 \mathrm{H}\right), 4.11-4.09(\mathrm{~m}, 4 \mathrm{H}), 4.06\left(\mathrm{~d},{ }^{4} J(\mathrm{H}, \mathrm{H})=2.4 \mathrm{~Hz}, 4 \mathrm{H}\right), 3.81-3.79(\mathrm{~m}$, $4 \mathrm{H}), 3.65-3.62(\mathrm{~m}, 4 \mathrm{H}), 3.58-3.55(\mathrm{~m}, 4 \mathrm{H}), 2.40\left(\mathrm{t},{ }^{4} J(\mathrm{H}, \mathrm{H})=2.4 \mathrm{~Hz}, 2 \mathrm{H}\right) .{ }^{13} \mathrm{C} \mathrm{NMR}(100$ $\mathrm{MHz}_{\mathrm{CDCl}}$ ): $\delta 154.21,126.64,125.08,114.53,105.64,79.66,74.80,70.56,69.66,69.06$, 67.80, 58.28. HRMS Calcd for $\mathrm{C}_{24} \mathrm{H}_{28} \mathrm{O}_{6}$ : 412.1886. Found: 412.1875. 
Scheme S7. Synthesis of DNP Diyne Derivative 9c.
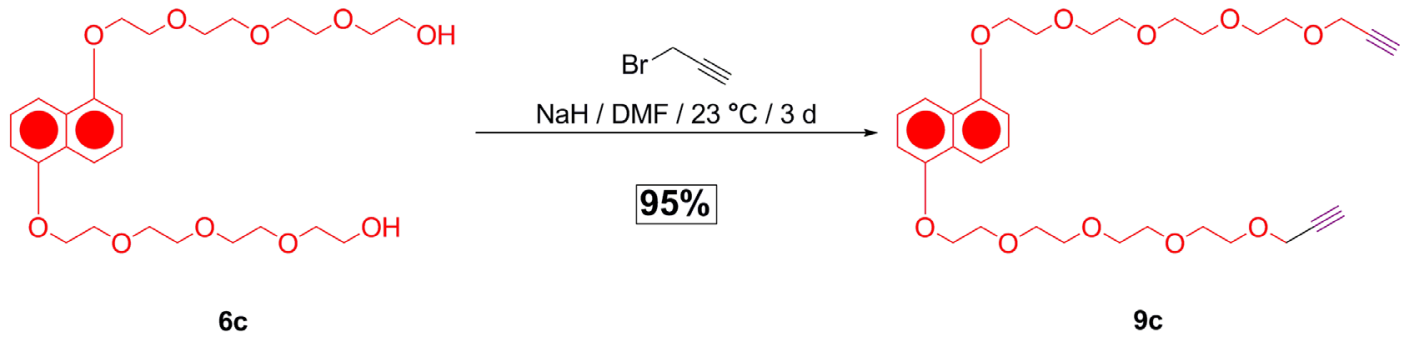

Diol 6c (1.28 g, $2.50 \mathrm{mmol})$ was dissolved in dry DMF $(25 \mathrm{~mL})$ and treated with $\mathrm{NaH}(156$ $\mathrm{mg}, 6.50 \mathrm{mmol})$. After the evolution of gas ceased ( $\sim 30 \mathrm{~min})$, propargyl bromide (1.8 $\mathrm{mL}$ of $80 \%$ wt solution in PhMe, $16.0 \mathrm{mmol}$ ) was added via a syringe. The mixture was stirred at $23{ }^{\circ} \mathrm{C}$ for $3 \mathrm{~d}$. $\mathrm{MeOH}(2.0 \mathrm{~mL})$ was added and the solvents were removed in vacuo. The crude brown oil was filtered through a plug of silica, eluting with $\mathrm{Me}_{2} \mathrm{CO}$, to provide pure 9c $(1.39 \mathrm{~g}, 95 \%)$ as a brown oil. 9c: MS (FAB+): $\mathrm{m} / \mathrm{z}$ (rel intensity) $=588\left(\mathrm{M}^{+}, 100 \%\right), 391$ (33). ${ }^{1} \mathrm{H}$ NMR $\left(500 \mathrm{MHz}, \mathrm{CDCl}_{3}\right): \delta 7.84\left(\mathrm{~d},{ }^{3} J(\mathrm{H}, \mathrm{H})=8.5 \mathrm{~Hz}, 2 \mathrm{H}\right), 7.33\left(\mathrm{t},{ }^{3} J(\mathrm{H}, \mathrm{H})=\right.$ $8.4 \mathrm{~Hz}, 2 \mathrm{H}), 6.83\left(\mathrm{~d},{ }^{3} J(\mathrm{H}, \mathrm{H})=8.4 \mathrm{~Hz}, 2 \mathrm{H}\right), 4.29-4.27(\mathrm{~m}, 4 \mathrm{H}), 4.17\left(\mathrm{~d},{ }^{4} J(\mathrm{H}, \mathrm{H})=2.4\right.$ $\mathrm{Hz}, 4 \mathrm{H}), 4.00-3.95$ (m, 4H), 3.80-3.78 (m, 4H), 3.70-3.67 (m, 4H), 3.67-3.63 (m, 16H), $2.41\left(\mathrm{t},{ }^{4} J(\mathrm{H}, \mathrm{H})=2.4 \mathrm{~Hz}, 2 \mathrm{H}\right) .{ }^{13} \mathrm{C}$ NMR $\left(125 \mathrm{MHz}, \mathrm{CDCl}_{3}\right): \delta 154.22,126.65,124.98$, $114.51,105.55,79.56,74.44,70.89,70.60,70.53,70.51,70.29,69.72,69.00,67.81,58.29$. HRMS Calcd for $\mathrm{C}_{32} \mathrm{H}_{44} \mathrm{O}_{10}$ : 588.2934. Found: 588.2942.

Scheme S8. Attempted Synthesis of Catenane $1 \mathrm{a} \cdot 4 \mathrm{PF}_{6}$.
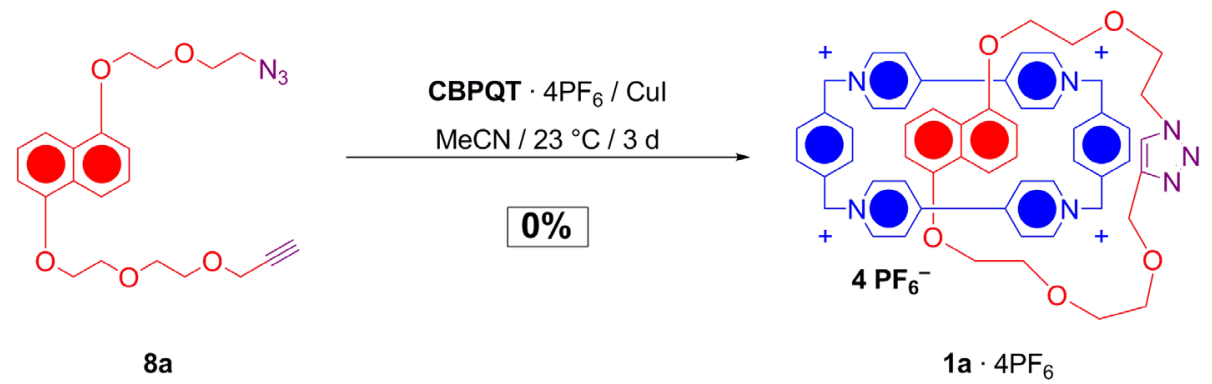

The DNP derivative 8a $(9.2 \mathrm{mg}, 0.024 \mathrm{mmol}), \mathbf{C B P Q T} \cdot 4 \mathrm{PF}_{6}(22.0 \mathrm{mg}, 0.02 \mathrm{mmol})$, and $\mathrm{CuI}(2.0 \mathrm{mg}, 0.01 \mathrm{mmol})$ were dissolved in $\mathrm{CD}_{3} \mathrm{CN}(0.6 \mathrm{~mL})$ and transferred into an NMR tube. The purple solution was left at $23{ }^{\circ} \mathrm{C}$ for $2 \mathrm{~d}$. After this time, ${ }^{1} \mathrm{H}$ NMR spectroscopy and FAB MS showed no sign of formation of $\mathbf{1 a} \cdot 4 \mathrm{PF}_{6}$. Performing the reaction with a $\mathrm{CuSO}_{4} \cdot 5 \mathrm{H}_{2} \mathrm{O} /$ ascorbic acid catalyst mixture gave identical results. 
Scheme S9. Synthesis of Catenane $1 \mathrm{c} \cdot 4 \mathrm{PF}_{6}$.

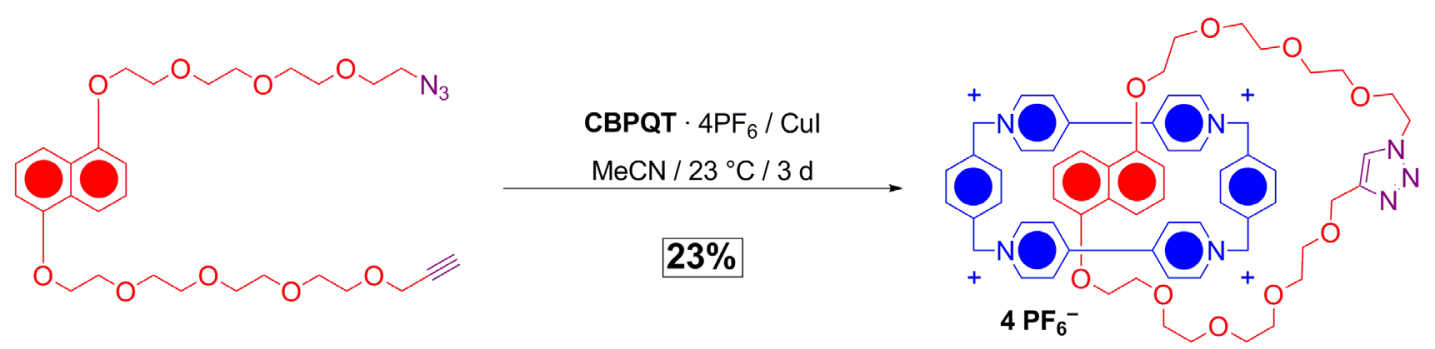

$8 c$

1c $4 \mathrm{PF}_{6}$

The DNP derivative 8c (34.5 mg, $0.06 \mathrm{mmol})$, CBPQT · 4PF 6 (44.0 mg, $0.04 \mathrm{mmol})$, and $\mathrm{CuI}(11.5 \mathrm{mg}, 0.06 \mathrm{mmol})$ were suspended in $\mathrm{CD}_{3} \mathrm{CN}(0.6 \mathrm{~mL})$ and transferred into an NMR tube. The purple solution was allowed to stand at $23{ }^{\circ} \mathrm{C}$ for $3 \mathrm{~d}$. After this time, the ${ }^{1} \mathrm{H}$ NMR spectroscopy showed peaks consistent with the presence of $1 \mathbf{c} \cdot 4 \mathrm{PF}_{6}$. The reaction mixture was filtered through a plug of silica (gradient elution: $\mathrm{Me}_{2} \mathrm{CO} \rightarrow 2 \%$ solution of $\mathrm{NH}_{4} \mathrm{PF}_{6}$ in $\mathrm{Me}_{2} \mathrm{CO}$ ). Purple fractions were collected, concentrated to a minimum volume and the crude catenane $1 \mathrm{c} \cdot 4 \mathrm{PF}_{6}$ was precipitated by the addition of $\mathrm{H}_{2} \mathrm{O}$. After filtration and drying, this crude material was analyzed and showed to contain CBPQT $\cdot 4 \mathrm{PF}_{6}$ and the desired [2]catenane $1 \mathbf{c} \cdot 4 \mathrm{PF}_{6}$. The crude mixture was dissolved in minimum amount of $\mathrm{Me}_{2} \mathrm{CO}$ and hexane was added dropwise until precipitation of CBPQT - 4 $\mathrm{PF}_{6}$ occurred. Removal of the solvent from the supernatant provided pure 1c . $4 \mathrm{PF}_{6}(15 \mathrm{mg}, 23 \%)$ as a purple solid, $\mathrm{mp} 199-201{ }^{\circ} \mathrm{C}$ with decomp. 1c $\cdot 4 \mathrm{PF}_{6}$ : $\mathrm{MS}$ (ESI): $\mathrm{m} / \mathrm{z}($ rel intensity $)=1530\left(\left[\mathbf{1 c} \cdot 3 \mathrm{PF}_{6}\right]^{+}, 8 \%\right), 692\left(\left[\mathbf{1 c} \cdot 2 \mathrm{PF}_{6}\right]^{2+}, 100\right), 413\left(\left[\mathbf{1 c} \cdot \mathrm{PF}_{6}\right]^{3+}\right.$, 45). ${ }^{1} \mathrm{H}$ NMR (500 MHz, DMF- $\left.d_{7}, 270 \mathrm{~K}\right): \delta 9.73\left(\mathrm{br} \mathrm{d}, 1 \mathrm{H}, \alpha-\mathrm{CBPQT}^{4+}-\mathrm{H}\right), 9.48\left(\mathrm{~d},{ }^{3} J\right.$ $\left.(\mathrm{H}, \mathrm{H})=6.5 \mathrm{~Hz}, 1 \mathrm{H}, \alpha-\mathrm{CBPQT}^{4+}-\mathrm{H}\right), 9.46\left(\mathrm{~d},{ }^{3} J(\mathrm{H}, \mathrm{H})=6.6 \mathrm{~Hz}, 1 \mathrm{H}, \alpha-\mathrm{CBPQT}^{4+}-\mathrm{H}\right), 9.44-$ $9.34\left(\mathrm{br} \mathrm{m}, 2 \mathrm{H}, \alpha-\mathrm{CBPQT}^{4+}-\mathrm{H}\right), 9.32\left(\mathrm{~d},{ }^{3} J(\mathrm{H}, \mathrm{H})=6.8 \mathrm{~Hz}, 1 \mathrm{H}, \alpha-\mathrm{CBPQT}^{4+}-\mathrm{H}\right), 9.28\left(\mathrm{~d},{ }^{3} J\right.$ $\left.(\mathrm{H}, \mathrm{H})=6.7 \mathrm{~Hz}, 1 \mathrm{H}, \alpha-\mathrm{CBPQT}^{4+}-\mathrm{H}\right), 8.81\left(\mathrm{br} \mathrm{s}, 1 \mathrm{H}, \alpha-\mathrm{CBPQT}^{4+}-\mathrm{H}\right), 8.31-8.09(\mathrm{~m}, 8 \mathrm{H}, \beta-$ $\mathrm{CBPQT}^{4+}-\mathrm{H}$ ), 7.85 (br m, 2H, aryl-CBPQT ${ }^{4+}-\mathrm{H}$ ), 7.82 (br s, 2H, aryl-CBPQT $\left.{ }^{4+}-\mathrm{H}\right), 7.72$ (br s, $2 \mathrm{H}$, aryl-CBPQT $\left.{ }^{4+}-\mathrm{H}\right), 7.62\left(\right.$ br s, $2 \mathrm{H}$, aryl-CBPQT $\left.{ }^{4+}-\mathrm{H}\right), 7.46(\mathrm{~s}, 1 \mathrm{H}$, triazole- $\mathrm{H})$, $6.29\left(\mathrm{~d},{ }^{3} J(\mathrm{H}, \mathrm{H})=7.9 \mathrm{~Hz}, 1 \mathrm{H}, \mathrm{DNP}\right.$ aryl-H $\left.o-\mathrm{O}\right), 6.10-5.91(\mathrm{~m}, 11 \mathrm{H}$, DNP aryl-H $o-\mathrm{O}$, DNP aryl-H $m-\mathrm{O}$, and $\mathrm{CBPQT}^{4+}$ benzyl-H), 4.18-2.69 (m, 32H), 3.03 (s, 2H, propargylH), $2.54\left(\mathrm{~d},{ }^{3} J(\mathrm{H}, \mathrm{H})=7.7 \mathrm{~Hz}, 1 \mathrm{H}, \mathrm{DNP}\right.$ aryl-H $\left.p-\mathrm{O}\right), 2.53\left(\mathrm{~d},{ }^{3} J(\mathrm{H}, \mathrm{H})=8.0 \mathrm{~Hz}, 1 \mathrm{H}\right.$, DNP 
aryl-H p-O). HRMS Calcd for $\mathrm{C}_{65} \mathrm{H}_{73} \mathrm{~N}_{7} \mathrm{O}_{9} \mathrm{P}_{2} \mathrm{~F}_{12}\left(\left[1 \mathrm{c} \cdot 2 \mathrm{PF}_{6}\right]^{2+}\right)$ : 692.7389. Found: 692.7350 .

Scheme S10. Attempted Synthesis of Catenane $2 \mathrm{a} \cdot 4 \mathrm{PF}_{6}$.

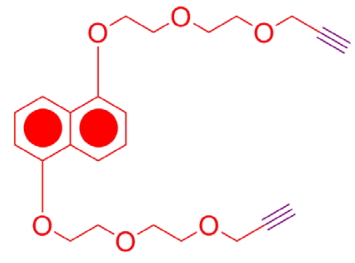

$9 a$

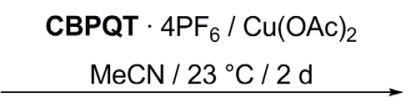

$0 \%$

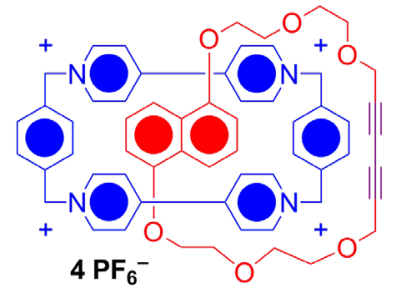

$2 \mathrm{a} \cdot 4 \mathrm{PF}_{6}$

The DNP derivative 9a (50.0 mg, $0.12 \mathrm{mmol})$, CBPQT $\cdot 4 \mathrm{PF}_{6}(44.0 \mathrm{mg}, 0.04 \mathrm{mmol})$, and $\mathrm{Cu}(\mathrm{OAc})_{2} \cdot \mathrm{H}_{2} \mathrm{O}(40.0 \mathrm{mg}, 0.20 \mathrm{mmol})$ were dissolved in $\mathrm{MeCN}(30 \mathrm{~mL})$. The deeply bluish-purple solution was stirred at $23{ }^{\circ} \mathrm{C}$ for $2 \mathrm{~d}$. The solvent was removed in vacuo, and the crude purple solid was subjected to column chromatography on silica $\left(\mathrm{Me}_{2} \mathrm{CO} \rightarrow 2 \%\right.$ solution of $\mathrm{NH}_{4} \mathrm{PF}_{6}$ in $\mathrm{Me}_{2} \mathrm{CO}$ ). Several fractions were collected, concentrated to a small volume, and precipitated by addition of cold $\mathrm{H}_{2} \mathrm{O}$. None of the materials isolated by filtration contained any evidence for the presence of $\mathbf{2 a} \cdot 4 \mathrm{PF}_{6}$ (by ${ }^{1} \mathrm{H}$ NMR spectroscopy and FAB MS). Additionally, although $\mathbf{2 a} \cdot 4 \mathrm{PF}_{6}$ was expected to be purple, all of the collected fractions were either colorless or pale yellow.

Scheme S11. Improved Synthesis of Catenane $2 \mathbf{b} \cdot 4 \mathrm{PF}_{6}$.

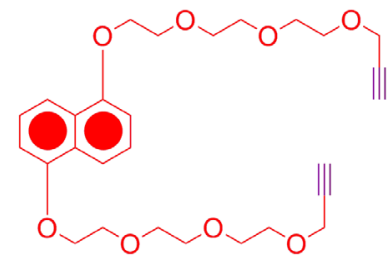

9b

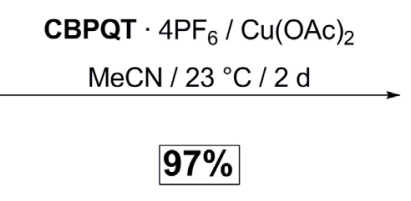

$97 \%$

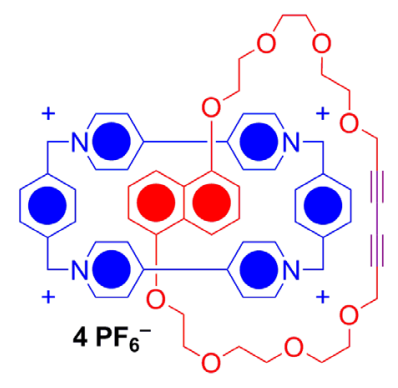

$\mathbf{2 b} \cdot 4 \mathrm{PF}_{6}$

The DNP derivative $\mathbf{9 b}(60.0 \mathrm{mg}, 0.12 \mathrm{mmol})$, CBPQT $\cdot 4 \mathrm{PF}_{6}(44.0 \mathrm{mg}, 0.04 \mathrm{mmol})$, and $\mathrm{Cu}(\mathrm{OAc})_{2} \cdot \mathrm{H}_{2} \mathrm{O}(40.0 \mathrm{mg}, 0.20 \mathrm{mmol})$ were dissolved in $\mathrm{MeCN}(30 \mathrm{~mL})$. The deeply bluish-purple solution was stirred at $23{ }^{\circ} \mathrm{C}$ for $2 \mathrm{~d}$. The solvent was removed in vacuo, and 
the crude purple solid was subjected to column chromatography on silica $\left(\mathrm{Me}_{2} \mathrm{CO} \rightarrow 2 \%\right.$ solution of $\mathrm{NH}_{4} \mathrm{PF}_{6}$ in $\mathrm{Me}_{2} \mathrm{CO}$ ). Purple fractions were collected and reduced to minimal volume. Cold $\mathrm{H}_{2} \mathrm{O}$ was added to precipitate the purple solid. After filtration and drying, 58 $\mathrm{mg}(97 \%)$ of $\mathbf{2 b} \cdot 4 \mathrm{PF}_{6}$ was obtained. The spectroscopic data for this sample were identical to one previously reported. ${ }^{\mathrm{S} 6}$ The amount of $\mathbf{2} \mathbf{b} \cdot 4 \mathrm{PF}_{6}$ prepared allowed the collection of ${ }^{13} \mathrm{C}$ NMR spectrum. 2b $\cdot 4 \mathrm{PF}_{6}:{ }^{13} \mathrm{C}$ NMR (125 MHz, $\left.\mathrm{CD}_{3} \mathrm{CN}\right): \delta 151.00,145.12,144.37$, $136.48,131.29,131.07,128.02,125.92,124.45,124.35,108.27,104.12,70.75,70.54$, $70.12,69.70,69.48,68.25,67.84,57.89$.

Scheme S12. Synthesis of Catenane $2 \mathrm{c} \cdot 4 \mathrm{PF}_{6}$.

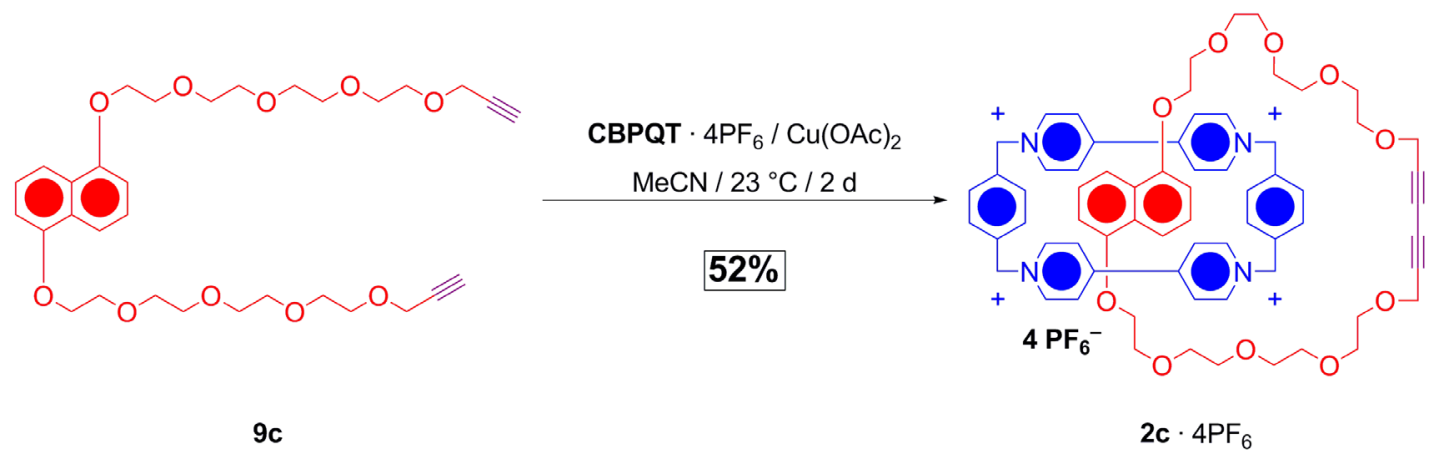

The DNP derivative 9c (72.0 mg, $0.12 \mathrm{mmol})$, CBPQT · 4PF 6 (44.0 mg, $0.04 \mathrm{mmol})$, and $\mathrm{Cu}(\mathrm{OAc})_{2} \cdot \mathrm{H}_{2} \mathrm{O}(40.0 \mathrm{mg}, 0.20 \mathrm{mmol})$ were dissolved in $\mathrm{MeCN}$ (30 mL). The deeply bluish-purple solution was stirred at $23{ }^{\circ} \mathrm{C}$ for $2 \mathrm{~d}$. The solvent was removed in vacuo, and the crude purple solid was subjected to column chromatography on silica $\left(\mathrm{Me}_{2} \mathrm{CO} \rightarrow 1 \%\right.$ solution of $\mathrm{NH}_{4} \mathrm{PF}_{6}$ in $\mathrm{Me}_{2} \mathrm{CO}$ ). Purple fractions were collected and concentrated to dryness. Cold $\mathrm{H}_{2} \mathrm{O}$ was added to precipitate the purple solid. After filtration and drying, this crude material was analyzed and showed to contain CBPQT $\cdot 4 \mathrm{PF}_{6}$ and the desired $\mathbf{2 c}$ - $4 \mathrm{PF}_{6}$. The crude mixture was dissolved in minimal amount of acetone and hexane was added dropwise until precipitation of CBPQT $\cdot 4 \mathrm{PF}_{6}$ occurred. Removal of the solvent from the supernatant provided pure $2 \mathrm{c} \cdot 4 \mathrm{PF}_{6}(35 \mathrm{mg}, 52 \%)$ as a purple solid, $\mathrm{mp}>230{ }^{\circ} \mathrm{C}$ without decomp. 2c $\cdot 4 \mathrm{PF}_{6}: \mathrm{MS}(\mathrm{ESI}): \mathrm{m} / z$ (rel intensity) $=1541\left(\left[\mathbf{2 c} \cdot 3 \mathrm{PF}_{6}\right]^{+}, 1 \%\right), 698$ $\left(\left[2 \mathrm{c} \cdot 2 \mathrm{PF}_{6}\right]^{2+}, 62\right), 563(100), 417\left(\left[2 \mathrm{c} \cdot \mathrm{PF}_{6}\right]^{3+}, 31\right) .{ }^{1} \mathrm{H} \mathrm{NMR}\left(500 \mathrm{MHz}, \mathrm{CD}_{3} \mathrm{CN}\right): \delta 9.01$ (br s, 2H, $\alpha-\mathrm{CBPQT}^{4+}-\mathrm{H}$ ), 8.86 (br s, 4H, $\alpha-\mathrm{CBPQT}^{4+}-\mathrm{H}$ ), 8.65 (br s, 2H, $\alpha-\mathrm{CBPQT}^{4+}-\mathrm{H}$ ), 8.02 (br s, 8H, $\left.\beta-\mathrm{CBPQT}^{4+}-\mathrm{H}\right), 7.34$ (br s, 8H, aryl-CBPQT $\left.{ }^{4+}-\mathrm{H}\right), 6.27\left(\mathrm{~d},{ }^{3} J(\mathrm{H}, \mathrm{H})=7.8\right.$ 
$\mathrm{Hz}, 2 \mathrm{H}, \mathrm{DNP}$ aryl-H $o-\mathrm{O}), 6.02\left(\mathrm{t},{ }^{3} J(\mathrm{H}, \mathrm{H})=8.0 \mathrm{~Hz}, 2 \mathrm{H}, \mathrm{DNP}\right.$ aryl-H $\left.m-\mathrm{O}\right), 5.74$ (br s, 8H, CBPQT ${ }^{4+}$ benzyl H), $4.31(\mathrm{~m}, 4 \mathrm{H}), 4.17(\mathrm{~m}, 4 \mathrm{H}), 4.02(\mathrm{~m}, 4 \mathrm{H}), 3.89(\mathrm{~m}, 4 \mathrm{H}), 3.85(\mathrm{~s}$, 4H, propargyl-H), $3.73(\mathrm{~m}, 2 \mathrm{H}), 3.56(\mathrm{~m}, 2 \mathrm{H}), 3.40(\mathrm{~m}, 2 \mathrm{H}), 3.19(\mathrm{~m}, 2 \mathrm{H}), 2.50$ (br s, $2 \mathrm{H}$, DNP aryl-H p-O). HRMS Calcd for $\mathrm{C}_{68} \mathrm{H}_{74} \mathrm{~N}_{4} \mathrm{O}_{10} \mathrm{P}_{2} \mathrm{~F}_{12}\left(\left[6 \cdot 2 \mathrm{PF}_{6}\right]^{2+}\right): 698.2350$. Found: 698.2326. 


\section{X-Ray Diffraction: Data Collection and Refinement Parameters}

[9a $\subset \mathbf{C B P Q T}] \cdot 4 \mathrm{PF}_{6}: \mathrm{C}_{60} \mathrm{H}_{60} \mathrm{~F}_{24} \mathrm{~N}_{4} \mathrm{O}_{6} \mathrm{P}_{4}$, crystal size $0.20 \times 0.20 \times 0.15 \mathrm{~mm}^{3}$, space group $P \overline{1}$, scan range $8.0<2 \theta<56.6^{\circ}, T=100(2) \mathrm{K}, a=10.163(3), b=12.669(4), c=$ 14.245(5) $\AA, \alpha=99.963(4), \beta=107.120(3), \gamma=112.522(3), V=1531.9(9) \AA^{3}, Z=1$, $\rho_{\text {calcd }}=1.640 \mathrm{gcm}^{-3}, \mu\left(\mathrm{MoK}_{\alpha}\right)=0.253 \mathrm{~mm}^{-1}, 7331$ unique reflections, of which 5083 were taken as observed [I>2 $\sigma(I)], R(F)=0.059, w R\left(F^{2}\right)=0.155$ (all data).

1b - $4 \mathrm{PF}_{6}: \mathrm{C}_{73} \mathrm{H}_{83} \mathrm{~F}_{24} \mathrm{~N}_{13} \mathrm{O}_{7} \mathrm{P}_{4}$, crystal size $0.60 \times 0.40 \times 0.05 \mathrm{~mm}^{3}$, space group $P \overline{1}$, scan range $7.8<2 \theta<53.1^{\circ}, T=100(2) \mathrm{K}, a=15.304(7), b=15.316(7), c=21.776(14) \AA, \alpha$ $=95.059(7), \beta=106.489(7), \gamma=116.953(5), V=4220(4) \AA^{3}, Z=2, \rho_{\text {calcd }}=1.444$ $\mathrm{gcm}^{-3}, \mu\left(\mathrm{MoK}_{\alpha}\right)=0.201 \mathrm{~mm}^{-1}, 17307$ unique reflections, of which 7251 were taken as observed $[I>2 \sigma(I)], R(F)=0.076, w R\left(F^{2}\right)=0.239$ (all data).

2b $\cdot 4 \mathrm{PF}_{6}: \mathrm{C}_{76} \mathrm{H}_{84} \mathrm{~F}_{24} \mathrm{~N}_{10} \mathrm{O}_{8} \mathrm{P}_{4}$, crystal size $0.50 \times 0.40 \times 0.20 \mathrm{~mm}^{3}$, space group $P \overline{1}$, scan range $8.4<2 \theta<59.7^{\circ}, T=100(2) \mathrm{K}, a=13.690(2), b=13.848(2), c=24.046(4) \AA, \alpha=$ 84.815(2), $\beta=85.732(2), \gamma=67.626(2), V=4194.3(11) \AA^{3}, Z=1, \rho_{\text {calcd }}=1.461 \mathrm{gcm}^{-3}$, $\mu\left(\mathrm{MoK}_{\alpha}\right)=0.203 \mathrm{~mm}^{-1}, 23132$ unique reflections, of which 17155 were taken as observed $[I>2 \sigma(I)], R(F)=0.076, w R\left(F^{2}\right)=0.222$ (all data).

2c $\cdot 4 \mathrm{PF}_{6}: \mathrm{C}_{80} \mathrm{H}_{92} \mathrm{~F}_{24} \mathrm{~N}_{13} \mathrm{O}_{10} \mathrm{P}_{4}$, crystal size $0.60 \times 0.30 \times 0.10 \mathrm{~mm}^{3}$, space group $P \overline{1}$, scan range $7.8<2 \theta<56.5^{\circ}, T=100(2) \mathrm{K}, a=14.021(3), b=14.121(3), c=22.210(5) \AA, \alpha=$ 94.628(2), $\beta=93.400(2), \gamma=93.554(3), V=4365.5(17) \AA^{3}, Z=2, \rho_{\text {calcd }}=1.470 \mathrm{gcm}^{-3}$, $\mu\left(\mathrm{MoK}_{\alpha}\right)=0.200 \mathrm{~mm}^{-1}, 20861$ unique reflections, of which 13530 were taken as observed $[I>2 \sigma(I)], R(F)=0.085, w R\left(F^{2}\right)=0.275$ (all data). 


\section{Supramolecular Organization of Crystallographically Characterized Materials}

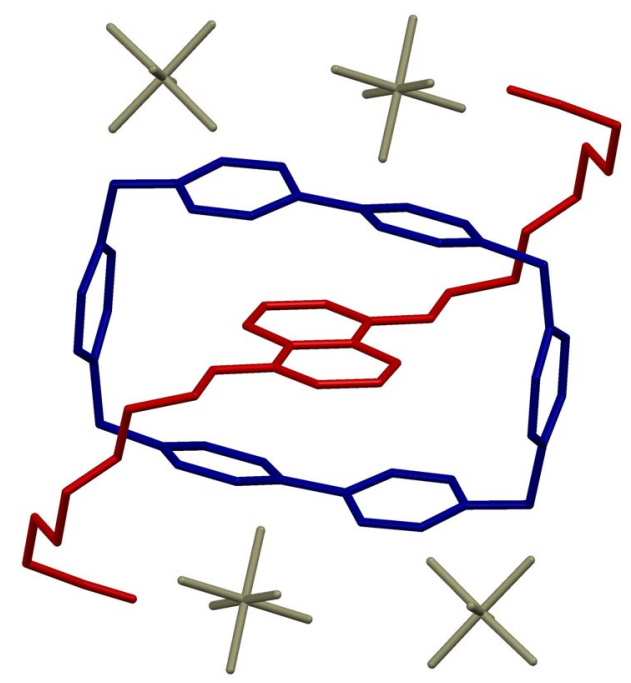

Figure S1. Unit cell contents of [2]pseudorotaxane $[9 \mathrm{a} \subset \mathrm{CBPQT}]^{4+}$. Donor rings shown in red, acceptor rings in blue, $\mathrm{PF}_{6}^{-}$counterions in gray. Aside from the remaining $\mathrm{PF}_{6}{ }^{-}$ counterions, hydrogen atoms and solvent molecules were omitted for clarity.

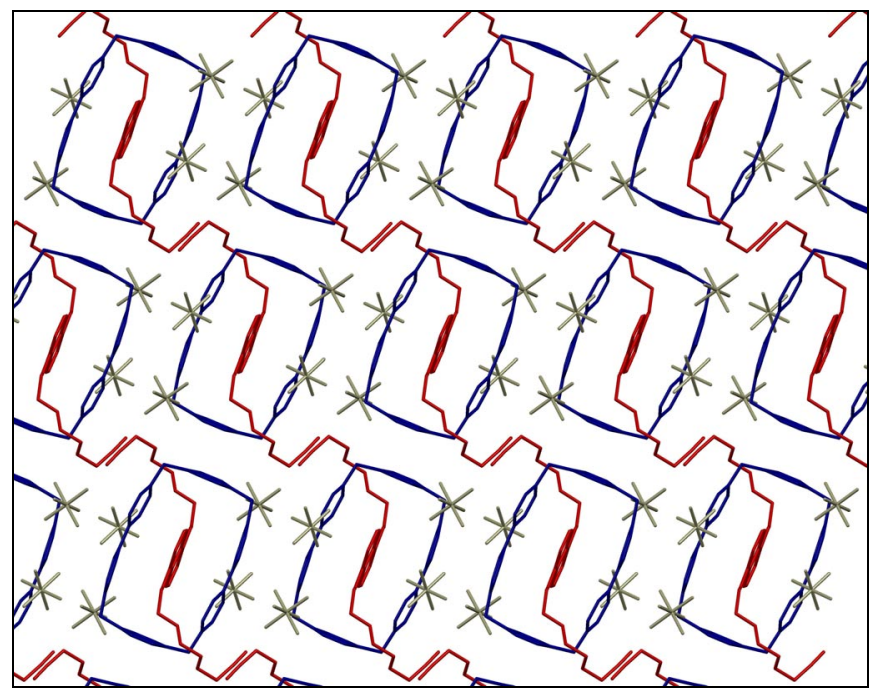

Figure S2. Section of the packing diagram of [2]pseudorotaxane $[\mathbf{9 a} \subset \mathbf{C B P Q T}]^{4+}$, viewed down the crystallographic $a$ axis. Donor rings shown in red, acceptor rings in blue. Aside from $\mathrm{PF}_{6}{ }^{-}$counterions, hydrogen atoms and solvent molecules were omitted for clarity. 

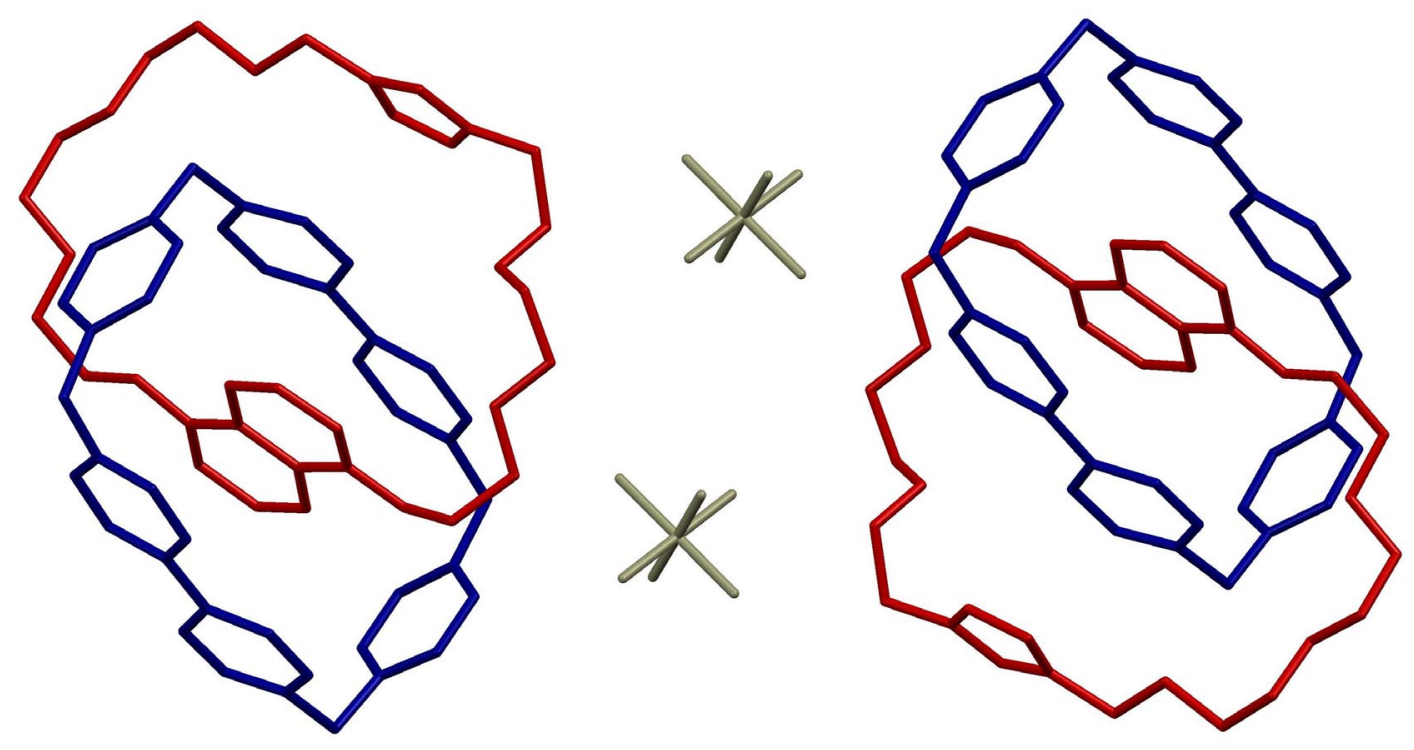

Figure S3. Unit cell contents of [2]catenane $\mathbf{1 b}^{4+}$. Donor rings shown in red, acceptor rings in blue, bridging $\mathrm{PF}_{6}{ }^{-}$counterions in gray. Aside from the remaining $\mathrm{PF}_{6}^{-}$counterions, hydrogen atoms and solvent molecules were omitted for clarity.

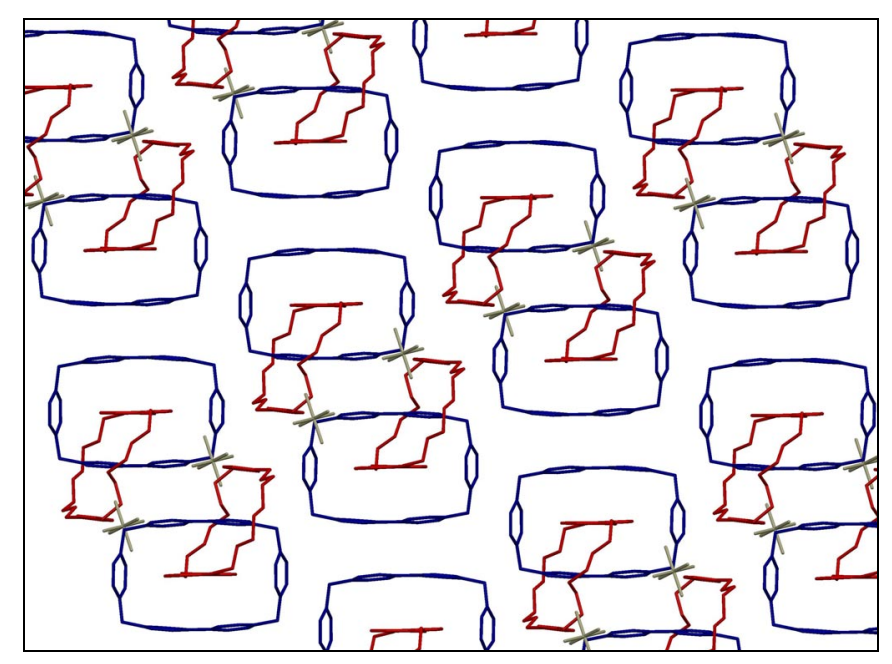

Figure S4. Section of the packing diagram of [2]catenane $1 \mathbf{b}^{4+}$. Donor rings shown in red, acceptor rings in blue. Aside from $\mathrm{PF}_{6}{ }^{-}$counterions, hydrogen atoms and solvent molecules were omitted for clarity. 


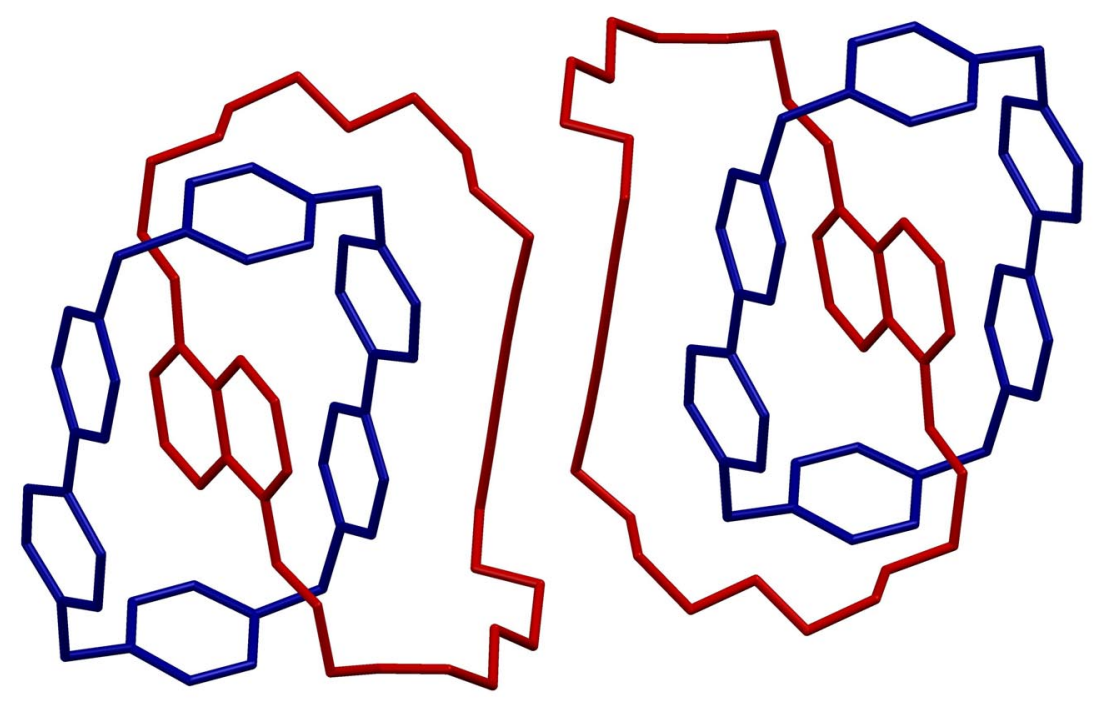

Figure S5. Unit cell contents of [2]catenane $\mathbf{2} \mathbf{b}^{4+}$. Donor rings shown in red, acceptor rings in blue. Aside from $\mathrm{PF}_{6}{ }^{-}$counterions, hydrogen atoms and solvent molecules were omitted for clarity.

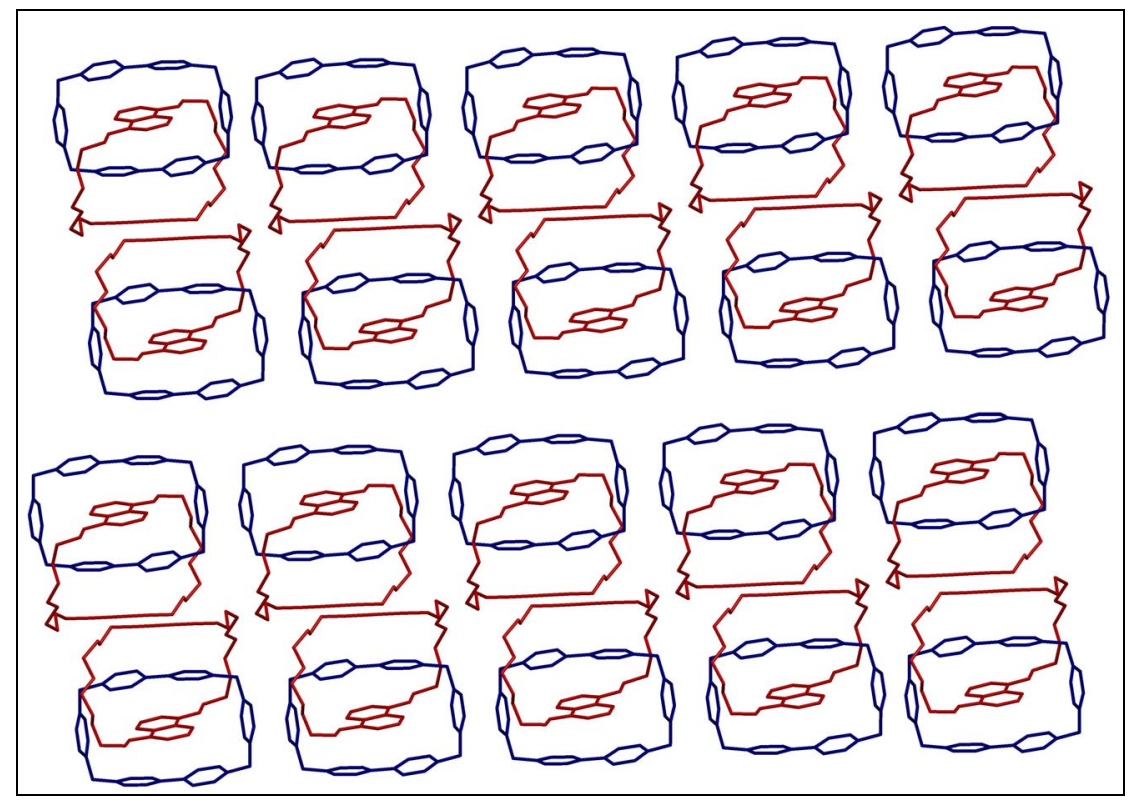

Figure S6. Section of the packing diagram of [2]catenane $\mathbf{2} \mathbf{b}^{4+}$. Donor rings shown in red, acceptor rings in blue. Aside from $\mathrm{PF}_{6}{ }^{-}$counterions, hydrogen atoms and solvent molecules were omitted for clarity. 

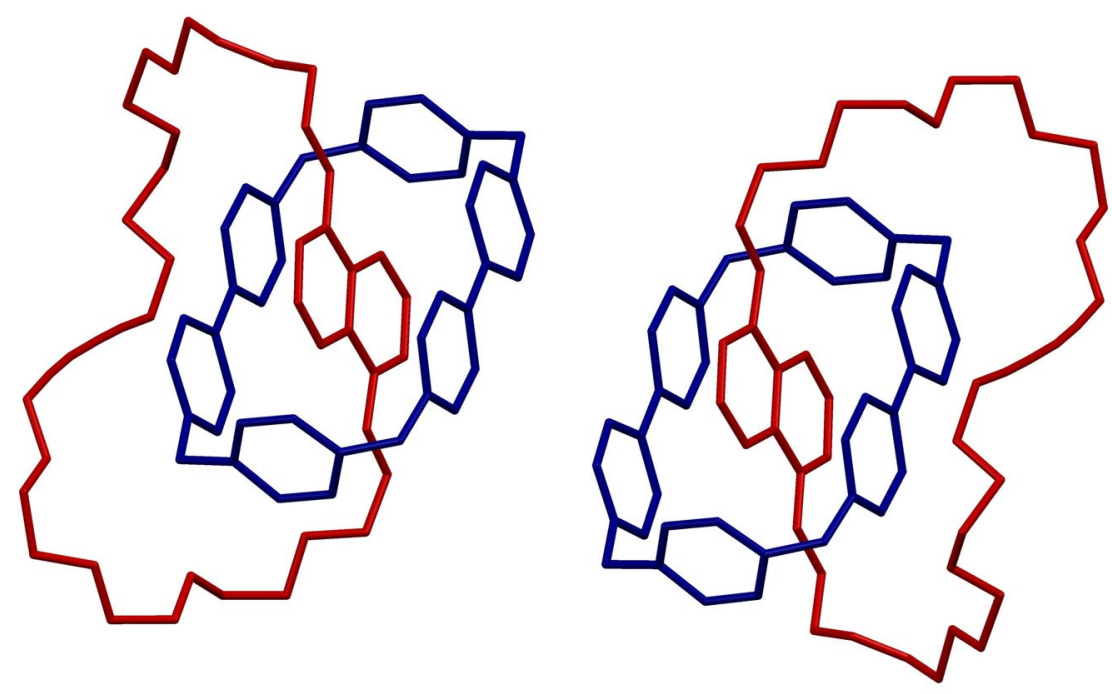

Figure S7. Unit cell contents of [2]catenane $\mathbf{2 c}^{4+}$. Donor rings shown in red, acceptor rings in blue. Aside from $\mathrm{PF}_{6}^{-}$counterions, hydrogen atoms and solvent molecules were omitted for clarity.

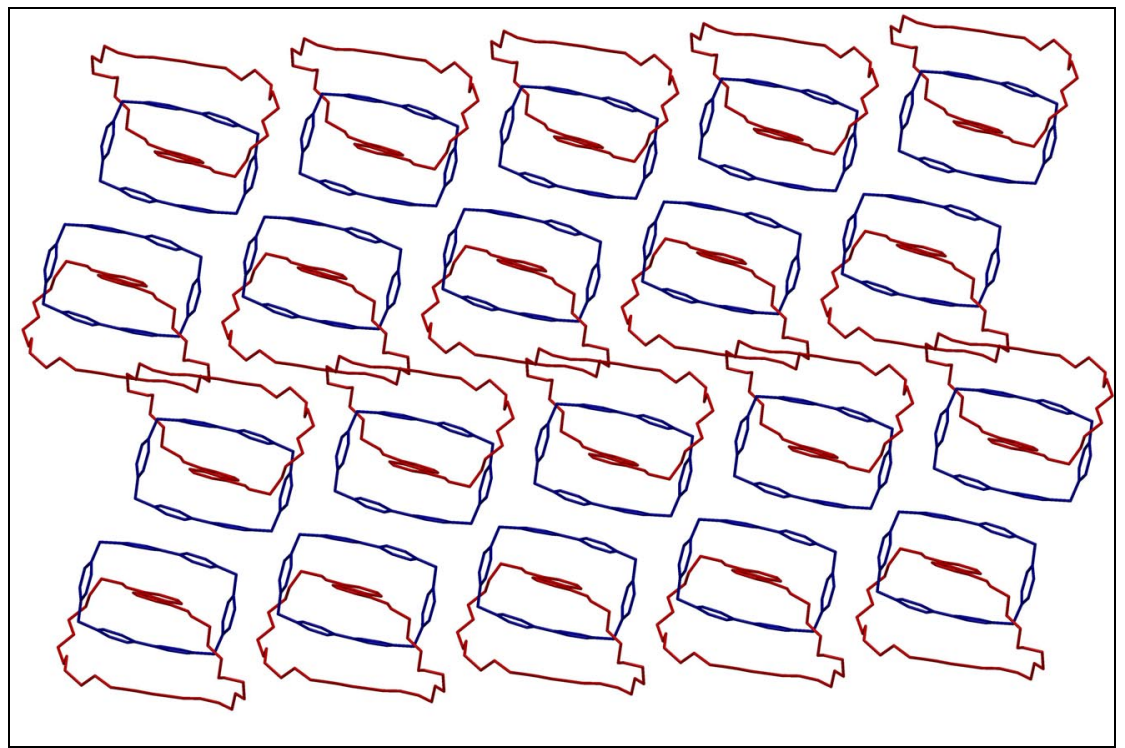

Figure S8. Section of the packing diagram of [2]catenane $\mathbf{2} \mathbf{c}^{4+}$. Donor rings shown in red, acceptor rings in blue. Aside from $\mathrm{PF}_{6}^{-}$counterions, hydrogen atoms and solvent molecules were omitted for clarity. 
Partial VT-NMR spectra of [2]catenanes $1 \mathrm{~b}, \mathrm{c} \cdot 4 \mathrm{PF}_{6}$ and $2 \mathrm{~b}, \mathrm{c} \cdot 4 \mathrm{PF}_{6}$

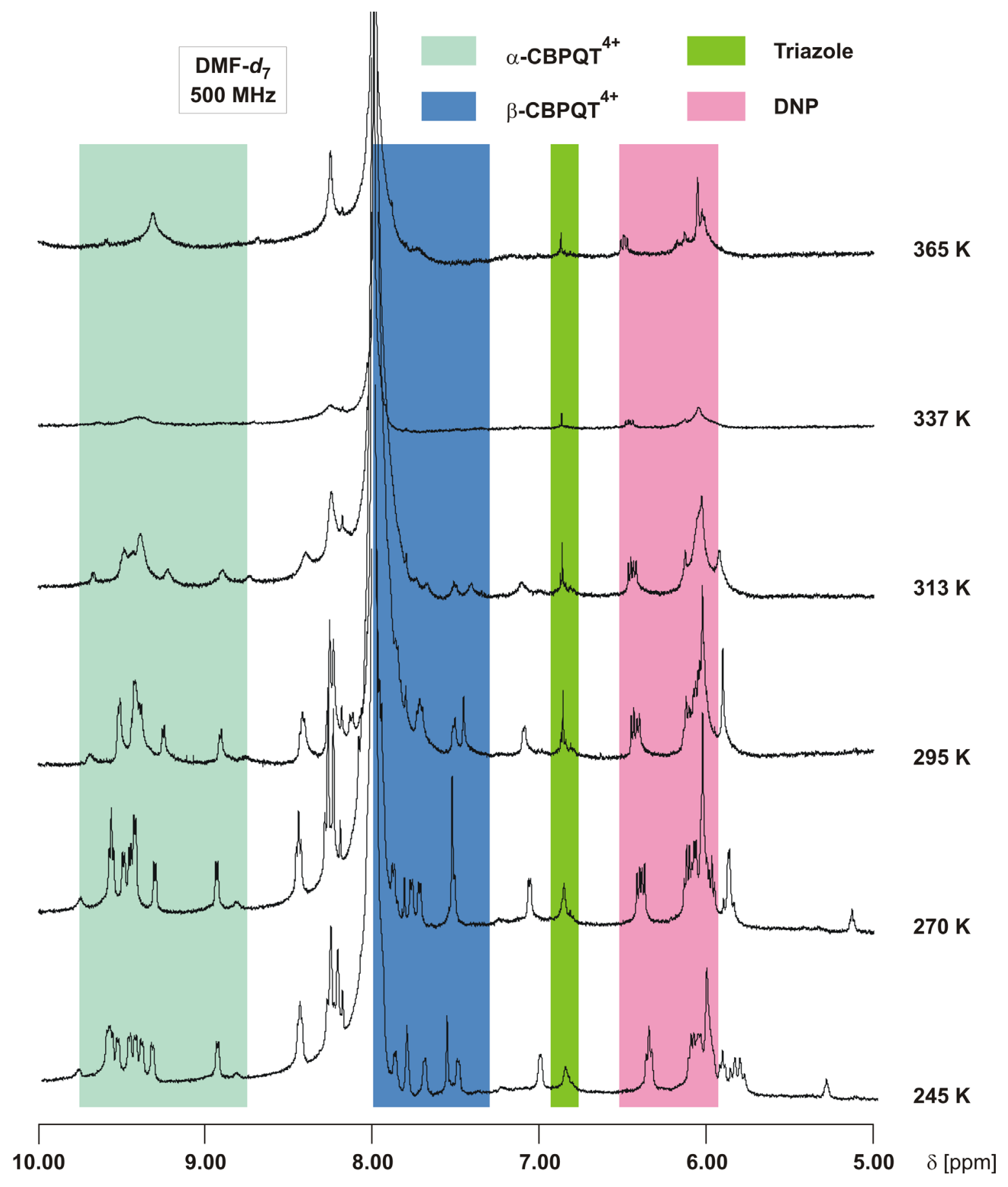

Figure S9. VT-NMR spectra of $\mathbf{1 b} \cdot 4 \mathrm{PF}_{6}\left(\mathrm{DMF}-d_{7}\right)$. 


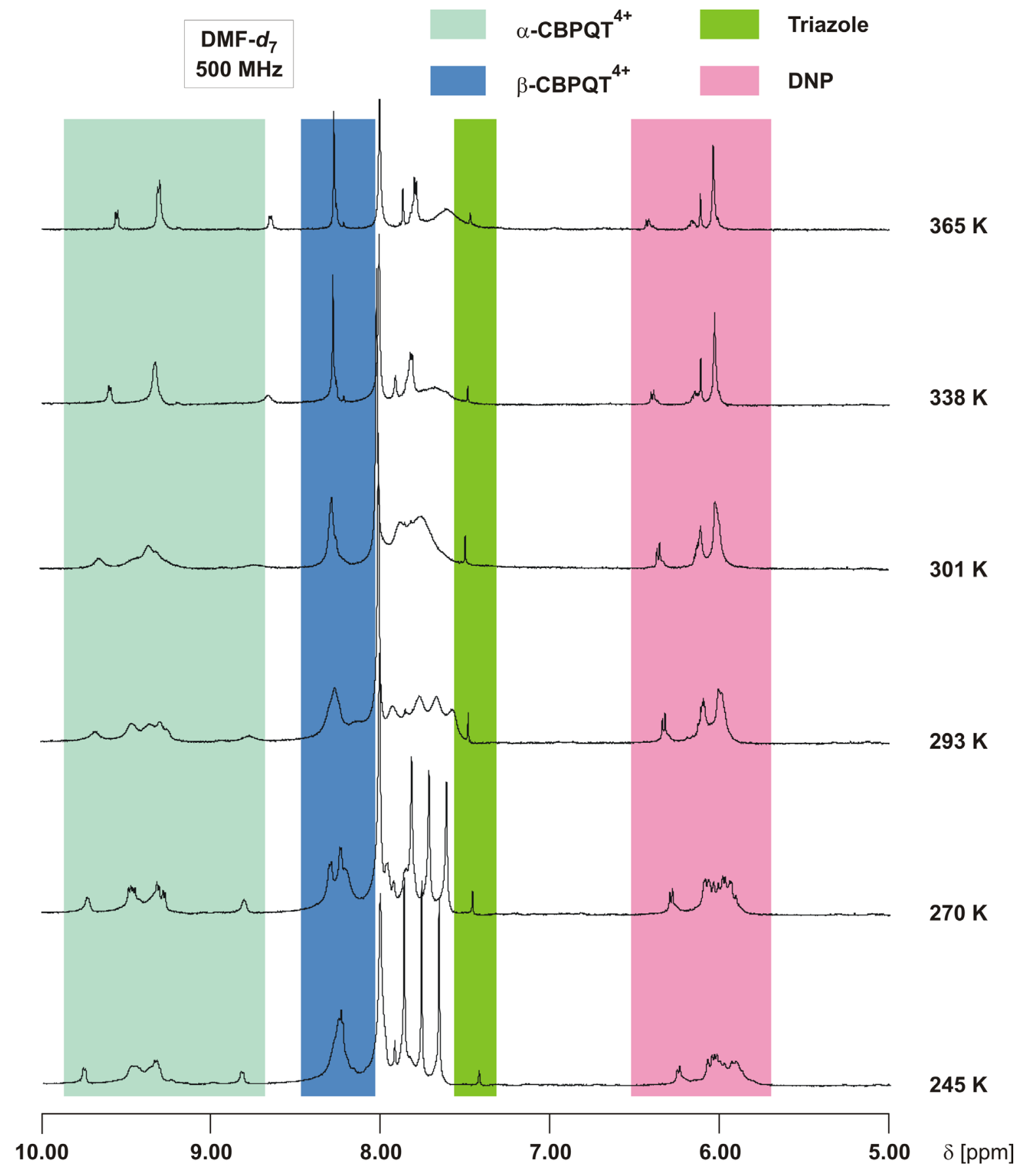

Figure S10. VT-NMR spectra of $1 \mathrm{c} \cdot 4 \mathrm{PF}_{6}\left(\mathrm{DMF}-d_{7}\right)$. 


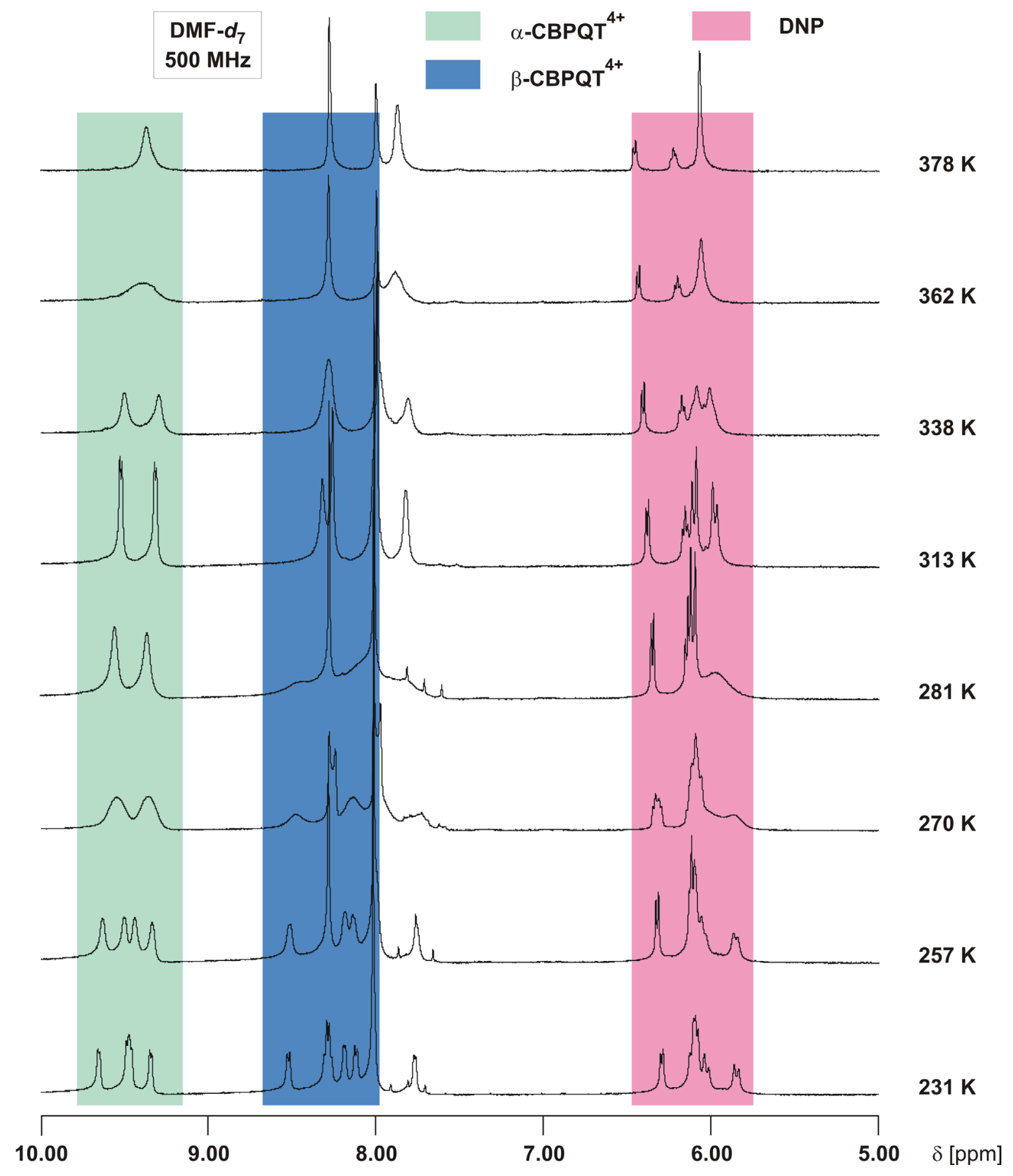

Figure S11. VT-NMR spectra of $\mathbf{2 b} \cdot 4 \mathrm{PF}_{6}\left(\mathrm{DMF}-d_{7}\right)$. 


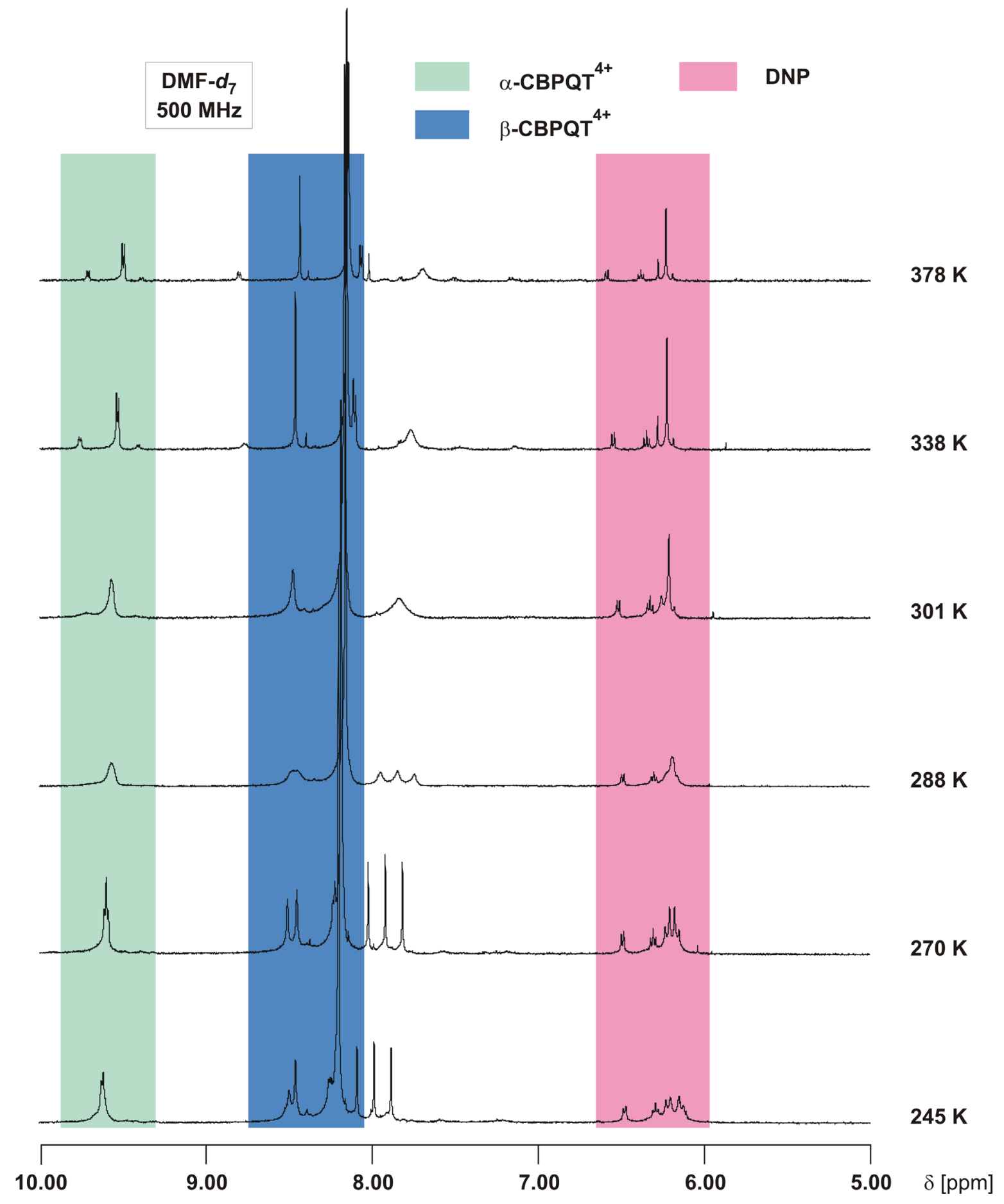

Figure S12. VT-NMR spectra of $2 \mathrm{c} \cdot 4 \mathrm{PF}_{6}\left(\mathrm{DMF}-d_{7}\right)$. 


\section{References}

(S1) Asakawa, M.; Dehaen, W.; L’abbé, G.; Menzer, S.; Nouwen, J.; Raymo, F. M.; Stoddart, J. F.; Williams, D. J. J. Org. Chem. 1996, 61, 9591-9595.

(S2) Amabilino, D. B.; Anelli, P.-L.; Ashton, P. R.; Brown, G. R.; Córdova, E.; Godínez, L. A.; Hayes, W.; Kaifer, A. E.; Philp, D.; Slawin, A. M. Z.; Spencer, N.; Stoddart, J. F.; Tolley, M. S.; Williams, D. J. J. Am. Chem. Soc. 1995, 117, 1114211170.

(S3) Ashton, P. R.; Huff, J.; Menzer, S.; Parsons, I. W.; Preece, J. A.; Stoddart, J. F.; Tolley, M. S.; White, A. J. P.; Williams, D. J. Chem. Eur. J. 1996, 2, 31-44.

(S4) Collier, C. P.; Jeppesen, J. O.; Liu, Y.; Perkins, J.; Wong, E. W.; Heath, J. R.; Stoddart, J. F. J. Am. Chem. Soc. 2001, 123, 12632-12641.

(S5) Liu, Y.; Saha, S.; Vignon, S. A.; Flood, A. H.; Stoddart, J. F. Synthesis 2005, 34373455 .

(S6) Miljanić, O. Š.; Dichtel, W. R.; Mortezaei, S.; Stoddart, J. F. Org. Lett. 2006, 8, $4835-4838$.

\section{Full References from the Manuscript}

(6j) Green, J. E.; Choi, J. W.; Boukai, A.; Bunimovich, Y.; Johnston-Halprin, E.; DeIonno, E.; Luo, Y.; Sheriff, B. A.; Xu, K.; Shin, Y. S.; Tseng, H.-R.; Stoddart, J. F.; Heath, J. R. Nature 2007, 445, 414-417.

(11c) Anelli, P.-L. Masumi Asakawa, M.; Ashton, P. R.; Bissell, R. A.; Clavier, G.; Górski, R.; Kaifer, A. E.; Langford, S. J.; Mattersteig, G.; Menzer, S.; Philp, D.; Slawin, A. M. Z.; Spencer, N.; Stoddart, J. F.; Tolley, M. S.; Williams, D. J. Chem.-Eur. J. 1997, 3, 1113-1135. 
(12c) Anelli, P.-L.; Ashton, P. R.; Ballardini, R.; Balzani, V.; Delgado, M.; Gandolfi, $M$. T.; Goodnow, T. T.; Kaifer, A. E.; Philp, D.; Pietraszkiewicz, M.; Prodi, L.; Reddington, M. V.; Slawin, A. M. Z.; Spencer, N.; Stoddart, J. F.; Vicent, C.; Williams, D. J. J. Am. Chem. Soc. 1992, 114, 193-218.

(14) Ashton, P. R.; Brown, C. L.; Chrystal, E. J. T.; Goodnow, T. T.; Kaifer, A. E.; Parry, K. P.; Philp, D.; Slawin, A. M. Z.; Spencer, N.; Stoddart, J. F.; Williams, D. J. J. Chem. Soc., Chem. Commun. 1991, 634-639.

(15c) Asakawa, M.; Ashton, P. R.; Balzani, V.; Credi, A.; Hamers, C.; Mattersteig, G.; Montalti, M.; Shipway, A. N.; Spencer, N.; Stoddart, J. F.; Tolley, M. S.; Venturi, M.; White, A. J. P.; Williams D. J. Angew. Chem. Int. Ed. 1998, 37, 333-337.

(30a) Amabilino, D. B.; Anelli, P.-L.; Ashton, P. R.; Brown, G. R.; Córdova, E.; Godínez, L. A.; Hayes, W.; Kaifer, A. E.; Philp, D.; Slawin, A. M. Z.; Spencer, N.; Stoddart, J. F.; Tolley, M. S.; Williams, D. J. J. Am. Chem. Soc. 1995, 117, 1114211170 .

(33) Asakawa, M.; Ashton, P. R.; Boyd, S. E.; Brown, C. L.; Gillard, R. E.; Kocian, O.; Raymo, F. M.; Stoddart, J. F.; Tolley, M. S.; White, A. J. P.; Williams, D. J. J. Org. Chem. 1997, 62, 26-37.

(48b) Ashton, P. R.; Balzani, V.; Credi, A.; Kocian, O.; Pasini, D.; Prodi, L.; Spencer, N.; Stoddart, J. F.; Tolley, M. S.; Venturi, M.; White, A. J. P.; Williams, D. J. Chem.Eur. J. 1998, 4, 590-607.

(49a) Liu, Y.; Flood, A. H.; Bonvallett, P. A.; Vignon, S. A.; Northrop, B. H.; Tseng, H. R.; Jeppesen, J. O.; Huang, T. J.; Brough, B.; Baller, M.; Magonov, S.; Solares, S. D.; Goddard, W. A.; Ho, C. M.; Stoddart, J. F. J. Am. Chem. Soc. 2005, 127, $9745-$ 9759.

(49b) Huang, T. J.; Brough, B.; Ho, C.-M.; Liu, Y.; Flood, A. H.; Bonvallet, P. A.; Tseng, H.-R.; Stoddart, J. F.; Baller, M.; Magonov, S. Appl. Phys. Lett. 2004, 85, 5391-5393. 